\title{
ELEVATORS FOR DEGENERATIONS OF PEL STRUCTURES
}

\author{
KAI-WEN LAN
}

\begin{abstract}
We show that the maximal rank of mixed characteristic degenerations of abelian varieties parameterized by a PEL-type Shimura variety are the same as the maximal rank of equicharacteristic zero degenerations (of abelian varieties parameterized by the same Shimura variety). As a byproduct, we obtain a simple proof of Yasuo Morita's conjecture in 1975 that an abelian variety with additional structures parameterized by a compact PEL-type Shimura variety has potential good reductions everywhere.
\end{abstract}

\section{Introduction}

All known integral models of toroidal compactifications of PEL-type Shimura varieties have natural stratifications characterized by the degeneration patterns of the universal abelian schemes. It is common wishful thinking that the (better understood) characteristic zero story should give us a fairly suggestive picture about the boundary stratification in general, including cases of bad reduction.

In this article, we shall partially justify this wishful thinking (for all PEL-type Shimura varieties at once) by showing (at least) the ranks (of torus parts of degenerations) match in all characteristics. Let us be more precise about this statement.

Let $\mathcal{O}$ be an order in a semisimple algebra, finite dimensional over $\mathbb{Q}$, together with a positive involution ${ }^{\star}$. By a PEL-type $\mathcal{O}$-lattice $\left(L,\langle\cdot, \cdot\rangle, h_{0}\right)$ (as in $[10$, Definition 1.2.1.3]), we mean the following data:

(1) An $\mathcal{O}$-lattice, namely a $\mathbb{Z}$-lattice $L$ with the structure of an $\mathcal{O}$-module.

(2) An alternating pairing $\langle\cdot, \cdot\rangle: L \times L \rightarrow \mathbb{Z}(1)$ satisfying $\langle b x, y\rangle=\left\langle x, b^{\star} y\right\rangle$ for any $x, y \in L$ and $b \in \mathcal{O}$, together with an $\mathbb{R}$-algebra homomorphism $h_{0}: \mathbb{C} \rightarrow$ $\operatorname{End}_{\mathcal{O}} \underset{\mathbb{Z}}{\otimes}(L \underset{\mathbb{Z}}{\otimes} \mathbb{R})$ satisfying $\left\langle h_{0}(z) x, y\right\rangle=\left\langle x, h_{0}\left(z^{c}\right) y\right\rangle$ for any $x, y \in L$ and $z \in \mathbb{C}$, and satisfying $(2 \pi \sqrt{-1})^{-1}\left\langle x, h_{0}(\sqrt{-1}) x\right\rangle>0$ for any nonzero $x \in L$. (In [10, Definition 1.2.1.3] $h_{0}$ was denoted by $h$. )

The datum of $\left(\mathcal{O}, \star, L,\langle\cdot, \cdot\rangle, h_{0}\right)$ defines a group functor $\mathrm{G}$ over $\operatorname{Spec}(\mathbb{Z})$ (as in [10, Definition 1.2.1.5]), and defines the reflex field $F_{0}$ (as in [10, Definition 1.2.5.4]). Let $\mathcal{H}$ be a neat open compact subgroup of $\mathrm{G}(\hat{\mathbb{Z}})$ (as in $[15,0.6]$ or $[10$, Definition 1.4.1.8]). Then the data of $\left(\mathcal{O}, \star, L,\langle\cdot, \cdot\rangle, h_{0}\right)$ and $\mathcal{H}$ define a moduli problem $\mathrm{M}_{\mathcal{H}}$ over $\operatorname{Spec}\left(F_{0}\right)$ by [10, Definition 1.4.1.4] (with $\square=\emptyset$ and so that $\mathcal{O}_{F_{0},(\square)}=F_{0}$ there), parameterizing tuples $\left(A, \lambda, i, \alpha_{\mathcal{H}}\right)$ over schemes $S$ over $\operatorname{Spec}\left(F_{0}\right)$ of the following form:

(1) $A \rightarrow S$ is an abelian scheme.

(2) $\lambda: A \rightarrow A^{\vee}$ is a polarization.

(3) $i: \mathcal{O} \hookrightarrow \operatorname{End}_{S}(A)$ is an $\mathcal{O}$-endomorphism structure as in [10, Definition 1.3.3.1].

Received by the editors April 13, 2011. Revision received May 16, 2011.

1991 Mathematics Subject Classification. Primary 11G18, 14G35; Secondary 11G15, 14 D06. 
(4) $\underline{\operatorname{Lie}}_{A / S}$ with its $\mathcal{O} \underset{\mathbb{Z}}{\otimes} \mathbb{Q}$-module structure given naturally by $i$ satisfies the determinantal condition in $\left[10\right.$, Definition 1.3.4.2] given by $\left(L \underset{\mathbb{Z}}{\otimes} \mathbb{R},\langle\cdot, \cdot\rangle, h_{0}\right)$.

(5) $\alpha_{\mathcal{H}}$ is an (integral) level- $\mathcal{H}$ structure of $(A, \lambda, i)$ of type $(L \underset{\mathbb{Z}}{\otimes} \hat{\mathbb{Z}},\langle\cdot, \cdot\rangle)$ as in [10, Definition 1.3.7.8].

By [10, Theorem 1.4.1.12 and Corollary 7.2.3.10], $\mathrm{M}_{\mathcal{H}}$ is representable by a (smooth) quasi-projective scheme over $\operatorname{Spec}\left(F_{0}\right)$ under the assumption that $\mathcal{H}$ is neat.

Consider the set $\mathrm{X}=\mathrm{G}(\mathbb{R}) h_{0}$ of $\mathrm{G}(\mathbb{R})$-conjugates $h: \mathbb{C} \rightarrow \operatorname{End}_{\mathcal{O}} \underset{\mathbb{Z}}{\otimes}(L \underset{\mathbb{Z}}{\otimes} \mathbb{R})$ of the polarization $h_{0}: \mathbb{C} \rightarrow \operatorname{End}_{\mathcal{O} \underset{\mathbb{Z}}{\otimes} \mathbb{R}}(L \underset{\mathbb{Z}}{\otimes} \mathbb{R})$. Then it is well known (see [9, Section 8] or $\left[11\right.$, Section 2]) that there exists a quasi-projective variety $\mathrm{Sh}_{\mathcal{H} \text {,alg }}$ over $\mathbb{C}$, together with a canonical open and closed immersion $\mathrm{Sh}_{\mathcal{H} \text {,alg }} \hookrightarrow \mathrm{M}_{\mathcal{H}} \underset{F_{0}}{\otimes \mathbb{C}}$ when $\mathcal{H}$ is neat, such that the double-coset space $\mathrm{Sh}_{\mathcal{H}}:=\mathrm{G}(\mathbb{Q}) \backslash \mathrm{X} \times \mathrm{G}\left(\mathbb{A}^{\infty}\right) / \mathcal{H}$ can be identified with the analytification of $S h_{\mathcal{H}}$,alg. Moreover, if we denote by $S_{\mathcal{H}}$,can the schematic image

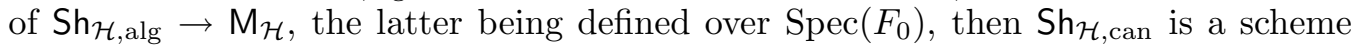

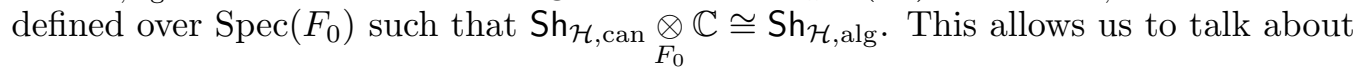
tuples $\left(A, \lambda, i, \alpha_{\mathcal{H}}\right)$ parameterized by $\mathrm{Sh}_{\mathcal{H} \text {,can }}$.

Consider semi-abelian schemes defined as in [4, Ch. I, Section 2]. By the rank of a fiber of a semi-abelian scheme, we mean the rank (as a free abelian group) of the character group of the torus part of any geometric fiber above the fiber. By a degeneration over a discrete valuation ring $R$ based at $\mathrm{Sh}_{\mathcal{H} \text {, can }}$, we mean a semiabelian scheme $A \rightarrow \operatorname{Spec}(R)$, together with a morphism $\operatorname{Spec}(\operatorname{Frac}(R)) \rightarrow \mathrm{Sh}_{\mathcal{H} \text {,can }}$, such that $A \otimes \operatorname{Frac}(R)$ is the pullback of the universal abelian variety over $\mathrm{Sh}_{\mathcal{H} \text {,can }}$ under $\operatorname{Spec}(\operatorname{Frac}(R)) \rightarrow \operatorname{Sh}_{\mathcal{H} \text {,can. }}$ By the rank of a degeneration $A \rightarrow \operatorname{Spec}(R)$ as above, we mean the rank of the special fiber of $A$. Note that we allow the rank to be zero, in which case $A$ is an abelian scheme over $\operatorname{Spec}(R)$.

Definition 1.1. For any characteristic $p \geq 0$ (of fields), we define $r_{p}$ to be the maximal rank among degenerations over discrete valuation rings $R$ based at $\mathrm{Sh}_{\mathcal{H} \text {,can }}$ with residue characteristic $p$. (The number $p$ is allowed to be zero.)

The invariant $r_{0}$ can be calculated explicitly, because of the following facts:

(1) The relation between the theory of degeneration and the algebraic construction of toroidal compactifications in the PEL-type cases is well understood. (See $[4,10]$.)

(2) The algebraic and analytic constructions of toroidal compactifications are known to be compatible over $\mathbb{C}$. (See [11] for an explanation using explicit identifications of theta functions. We will not need this fact in this article.)

(3) The analytic compactifications over $\mathbb{C}$ can be described group-theoretically. $($ See $[2,1,7,15]$.

Concretely, a degeneration of rank $r$ corresponds to an $\mathcal{O} \underset{\mathbb{Z}}{\otimes} \mathbb{Q}$-submodule of $L \underset{\mathbb{Z}}{\otimes} \mathbb{Q}$, totally isotropic under $\langle\cdot, \cdot\rangle$ and of dimension $r$ over $\mathbb{Q}$, determining a rational parabolic subgroup of $G \underset{\mathbb{Z}}{\otimes} \mathbb{Q}$. The relation between such parabolic subgroups of $G \underset{\mathbb{Z}}{\otimes} \mathbb{Q}$ and the rational boundary components of $X$ is well known. 
On the other hand, there is no obvious way to calculate the invariant $r_{p}$ for all characteristics $p \geq 0$. When $p$ is not a so-called bad prime in the sense of $[10$, Definition 1.4.1.1], the invariant $r_{p}$ agrees with $r_{0}$ because of the existence of a smooth toroidal compactification (with boundary stratified by smooth locally closed subschemes) over $\mathbb{Z}_{(p)}$ constructed using the theory of degeneration. (See [10, Theorem 6.4.1.1].) However, this does not cover the cases when $p$ is a bad prime. Our motivation for this article is to supply an argument independent of the residue characteristics. (We emphasize that the residue characteristic is irrelevant not only in the statement of the result, but also in the proof. No $p$ is special in this problem!)

The main result of this article.

Theorem 1.2. With the setting as above, we have $r_{0}=r_{p}$ for every $p \geq 0$.

Let us sketch the proof of Theorem 1.2 (and give an outline of the article) as follows. (In this sketch, for simplicity, we will abbreviate degenerations over discrete valuations based at $\mathrm{Sh}_{\mathcal{H} \text {,can }}$ as degenerations.)

Our first objective will be to prove Theorem 4.1, which asserts the inequality $r_{p} \leq r_{0}$ for every $p \geq 0$. This will follow from the existence of what we will call the elevators. Roughly speaking, for a given mixed characteristics degeneration of rank $r$, an elevator is a semi-abelian scheme over a noetherian normal domain, such that the given degeneration is a pullback of such a semi-abelian scheme, and such that at least one of its one characteristic zero fibers has rank equal to $r$. We will review the background terminologies in Sections 2.1 and 2.2, and give the precise definition of elevators in Section 2.3. We will then explain the construction of elevators in Section 3, using techniques developed in the construction of boundary charts of toroidal compactifications in $[4,10]$.

Once Theorem 4.1 is established, the special case $r_{p}=r_{0}=0$ for every $p \geq 0$ of the above inequality implies Yasuo Morita's original conjecture in [12]. (Note that $r_{p}=0$ for every $p \geq 0$ implies that all abelian varieties parameterized by $\mathrm{Sh}_{\mathcal{H} \text {,can }}$ have potential good reductions everywhere.) We shall explain this implication in Section 4.2, giving also a criterion of properness for integral models of PEL-type Shimura varieties in Theorem 4.9, with no assumption on the residue characteristics.

Once Theorem 4.1 is established, the special case $r_{p}=r_{0}=0$ for every $p \geq 0$ of the above inequality implies Yasuo Morita's original conjecture in [12]. (Note that $r_{p}=0$ for every $p \geq 0$ implies that all abelian varieties parameterized by $\mathrm{Sh}_{\mathcal{H} \text {,can }}$ have potential good reductions everywhere.) We shall explain this implication in Section 4.2, giving also a criterion of properness for integral models of PEL-type Shimura varieties in Theorem 4.9, with no assumption on the residue characteristics.

Then we will prove Theorem 5.1, which asserts the opposite inequality $r_{0} \leq r_{p}$ for every characteristic $p \geq 0$. The preparatory Proposition 5.2 (for proving Theorem 5.1) is the only step we use results in [10] not essentially known in [4], which allows us to choose an equicharacteristic zero degeneration of rank $r_{0}$, such that the abelian part of its (characteristic zero) special fiber extends to abelian schemes over discrete valuation rings of all possible mixed characteristics. (This extension uses Theorem 4.9, which we have just proved.) Starting with such mixed characteristics abelian schemes, by a construction similar to the one (of elevators) in Section 3, we obtain degenerations of rank $r_{0}$ over discrete valuation rings of all possible mixed characteristics, as desired.

Finally, Theorem 1.2 follows as a combination of Theorems 4.1 and 5.1. 
Following the referee's suggestion, we have included several commutative diagrams summarizing the constructions. Although these diagrams will not be logically needed in the proofs, we hope they will be helpful for understanding the arguments.

\section{Terminologies}

We shall follow [10, Notations and Conventions] unless otherwise specified. (The references to [10] uses the original numbering in the submitted thesis, but the reader is encouraged to consult the revision available on the author's website, sometimes with slightly modified numberings, for corrections and improvements.)

2.1. Degenerating families. Let $\mathcal{O}$ be as in Section 1 . We shall denote pullbacks of objects to rings or schemes by subscripts when there is no confusion.

Definition 2.1. Let $S$ be any normal locally noetherian scheme over $\operatorname{Spec}(\mathbb{Z})$. A degenerating family of type $(\mathrm{PE}, \mathcal{O})$ is a tuple $(A, \lambda, i)$ over $S$ such that:

(1) $A$ is a semi-abelian scheme over $S$.

(2) There exists an open dense subscheme $S_{1}$ of $S$ such that $A_{S_{1}}$ is an abelian scheme. In this case, there is a unique semi-abelian scheme $A^{\vee}$ (up to unique isomorphism), called the dual semi-abelian scheme of $A$, such that $A_{S_{1}}^{\vee}$ is the dual abelian scheme of $A_{S_{1}}$.

(3) $\lambda: A \stackrel{\sim}{\rightarrow} A^{\vee}$ is a group homomorphism that induces by restriction a polarization $\lambda_{S_{1}}$ of $A_{S_{1}}$.

(4) $i: \mathcal{O} \rightarrow \operatorname{End}_{S}(A)$ is a map that defines by restriction an $\mathcal{O}$-structure $i_{S_{1}}$ : $\mathcal{O} \rightarrow \operatorname{End}_{S_{1}}\left(A_{S_{1}}\right)$ of $\left(A_{S_{1}}, \lambda_{S_{1}}\right)$. (See [10, Definition 1.3.3.1].)

2.2. Theory of degeneration data. Let $\mathcal{O}$ be as above.

Let $R$ be a noetherian normal domain complete with respect to an ideal $I$, with $\operatorname{rad}(I)=I$ for convenience. Let $S:=\operatorname{Spec}(R), K:=\operatorname{Frac}(R), \eta:=\operatorname{Spec}(K)$ the generic point of $S, R_{0}:=R / I$, and $S_{0}:=\operatorname{Spec}\left(R_{0}\right)$. We shall denote the pullbacks to $\eta$ or $S_{0}$ by subscripts $\eta$ or 0 , respectively.

Definition 2.2 (cf. [10, Definition 5.1.1.4]). With notations and assumptions as above, the category $\mathrm{DEG}_{\mathrm{PE}, \mathcal{O}}^{\mathrm{split}}(R, I)$ has objects consisting of degenerating families $(A, \lambda, i)$ of type $(\mathrm{PE}, \mathcal{O})$ (over $S=\operatorname{Spec}(R))$ such that $A_{0}$ is an extension of an abelian scheme $B_{0}$ by a split torus $T_{0}$ over $S_{0}$.

By the theory of degeneration data for polarized abelian varieties in $[4$, Chs. II and III] (explained in [10, Ch. 4]), generalized by functoriality for polarized abelian varieties with endomorphisms (see [10, Section 5.1.1]), the so-called Mumford's construction induces an equivalence of categories

$$
\begin{aligned}
\mathrm{M}_{\mathrm{PE}, \mathcal{O}}^{\text {split }}(R, I): & \operatorname{DD}_{\mathrm{PE}, \mathcal{O}}^{\text {split }}(R, I) \rightarrow \operatorname{DEG}_{\mathrm{PE}, \mathcal{O}}^{\text {split }}(R, I) \\
& \left(B, \lambda_{B}, i_{B}, X, Y, \phi, c, c^{\vee}, \tau\right) \mapsto(A, \lambda, i)
\end{aligned}
$$

realizing $(A, \lambda, i)$ (up to isomorphism) as the image of an object in $\operatorname{DD}_{\mathrm{PE}, \mathcal{O}}^{\mathrm{split}}(R, I)$ given by the following data:

(1) An abelian scheme $B$ over $S$, a polarization $\lambda_{B}: B \rightarrow B^{\vee}$ of $B$, and an $\mathcal{O}$-endomorphism structure $i_{B}: \mathcal{O} \hookrightarrow \operatorname{End}_{S}(B)$ of $\left(B, \lambda_{B}\right)$. 
(2) Two $\mathcal{O}$-lattices $X$ and $Y$ of the same rank over $S$, with an $\mathcal{O}$-equivariant embedding $\phi: Y \rightarrow X$. (Here $X$ and $Y$ correspond, respectively, to the character groups of the torus parts of $A$ and $A^{\vee}$. We shall denote the actions of an element $b \in \mathcal{O}$ on $X$ and $Y$ by $i_{X}(b)$ and $i_{Y}(b)$, respectively. When the context is clear, we shall simply denote the actions by $b$.)

(3) Two $\mathcal{O}$-equivariant morphisms $c: X \rightarrow B^{\vee}$ and $c^{\vee}: Y \rightarrow B$ of group schemes over $S$, satisfying the compatibility $c \phi=\lambda_{B} c^{\vee}$. (By abuse of notation, we denote by $X$ and $Y$ the constant group schemes $X_{S}$ and $Y_{S}$ over $S$, respectively.)

(4) A trivialization of biextensions $\tau: \mathbf{1}_{Y \times X, \eta} \stackrel{\sim}{\rightarrow}\left(c^{\vee} \times c\right)^{*} \mathcal{P}_{B, \eta}^{\otimes-1}$ with symmetric pullback under $\operatorname{Id}_{Y} \times \phi: Y \times Y \rightarrow Y \times X$, satisfying the following conditions:

(a) Compatibility with $\mathcal{O}$-actions: For any $b \in \mathcal{O}$, we have a canonical identification of sections $\left(i_{Y}(b) \times \operatorname{Id}_{X}\right)^{*} \tau=\left(\operatorname{Id}_{Y} \times i_{X}\left(b^{\star}\right)\right)^{*} \tau$ under the canonical isomorphism $\left(i_{B}(b) \times \operatorname{Id}_{B^{\vee}}\right)^{*} \mathcal{P}_{B} \cong\left(\operatorname{Id}_{B} \times\left(i_{B}(b)\right)^{\vee}\right)^{*} \mathcal{P}_{B}$.

(b) Positivity: For any $y \in Y$ and $\chi \in X$, the trivialization $\tau(y, \chi)$ defines an isomorphism of invertible sheaves from $\left(c^{\vee}(y), c(\chi)\right)^{*} \mathcal{P}_{B, \eta}$ to $\mathbf{1}_{\eta}$. Under this isomorphism (which we again denote by $\tau(y, \chi)$ ), the canonical $R$-integral structure $\left(c^{\vee}(y), c(\chi)\right)^{*} \mathcal{P}_{B}$ of $\left(c^{\vee}(y), c(\chi)\right)^{*} \mathcal{P}_{B, \eta}$ determines an invertible $R$-submodule $I_{y, \chi}$ of $K$. Then the positivity condition is $I_{y, \phi(y)} \subset I$ for all nonzero $y$ in $Y$. (Clearly, $I_{0,0}=R$.)

We say that $\left(B, \lambda_{B}, i_{B}, X, Y, \phi, c, c^{\vee}, \tau\right)$ is the degeneration data of $(A, \lambda, i)$. The theory works even when $X$ and $Y$ are zero. (Then, by [4, Ch. I, 2.8], $A \cong B$ is an abelian scheme over $S$, and the positivity condition for $\tau$ is trivially verified.)

We shall suppress $I$ from the notation when it is clear from the context. (This is the case, for example, when $R$ is a discrete valuation ring.)

\subsection{Elevators for degenerations of PEL structures.}

Definition 2.3. Let $R^{\diamond}$ be a complete discrete valuation ring such that the characteristic of $K^{\diamond}:=\operatorname{Frac}\left(R^{\diamond}\right)$ is zero. Let $k^{\diamond}$ be the residue field of $R^{\diamond}$. Suppose $\left(A^{\diamond}, \lambda^{\diamond}, i^{\diamond}\right) \rightarrow \operatorname{Spec}\left(R^{\diamond}\right)$ is a degenerating family of type $(\mathrm{PE}, \mathcal{O})$. An elevator for $\left(A^{\diamond}, \lambda^{\diamond}, i^{\diamond}\right)$ is the following set of data:

(1) A complete discrete valuation ring $R$ with residue field $k$, together with a finite étale morphism $\operatorname{Spec}(R) \rightarrow \operatorname{Spec}\left(R^{\diamond}\right)$.

(2) A noetherian normal domain $\widetilde{R}$, together with a degenerating family $(\widetilde{A}, \widetilde{\lambda}, \widetilde{i})$ of type $(\mathrm{PE}, \mathcal{O})$ over $\widetilde{R}$.

(3) A morphism $\operatorname{Spec}(R) \rightarrow \operatorname{Spec}(\widetilde{R})$, together with an isomorphism

$$
\left(A^{\diamond}, \lambda^{\diamond}, i^{\diamond}\right) \underset{R^{\diamond}}{\otimes} R \stackrel{\sim}{\rightarrow}(\widetilde{A}, \widetilde{\lambda}, \widetilde{i}) \underset{\widetilde{R}}{\otimes} R .
$$

(In particular, the characteristic of $\widetilde{K}:=\operatorname{Frac}(\widetilde{R})$ is zero.)

(4) There is at least one (functorial) point $\operatorname{Spec}(\widetilde{k}) \rightarrow \operatorname{Spec}(\widetilde{R} \underset{\mathbb{Z}}{\otimes} \mathbb{Q})$ such that $\widetilde{A}_{\widetilde{k}}$ and $A_{k^{\diamond}}^{\diamond}$ have the same rank.

Note that an elevator is not quite a deformation. The ring $\widetilde{R}$ can stay the same when we replace $R^{\diamond}$ or $R$ with a much larger ring. The intuition is that an elevator should be part of something like a moduli space of degenerations of a similar pattern. 


\section{Construction of elevators}

Theorem 3.1. Let $R^{\diamond}$ and $\left(A^{\diamond}, \lambda^{\diamond}, i^{\diamond}\right)$ be as in Definition 2.3. Then there exists an elevator for $\left(A^{\diamond}, \lambda^{\diamond}, i^{\diamond}\right)$ satisfying the following additional conditions:

(1) The ring $\widetilde{R}$ is a noetherian normal domain over $R$, complete with respect to some ideal $\widetilde{I}$ such that $\operatorname{rad}(\widetilde{I})=\widetilde{I}$, and $(\widetilde{A}, \widetilde{\lambda}, \widetilde{i})$ is an object of $\operatorname{DEG}_{\mathrm{PE}, \mathcal{O}}^{\mathrm{split}}(\widetilde{R}, \widetilde{I})$.

(2) Suppose $\left(B, \lambda_{B}, i_{B}, X, Y, \phi, c, c^{\vee}, \tau\right)$ is the degeneration data associated with $\left(A^{\diamond}, \lambda^{\diamond}, i^{\diamond}\right) \underset{R^{\diamond}}{\otimes} R$, and suppose $\left(\widetilde{B}, \lambda_{\widetilde{B}}, i_{\widetilde{B}}, \widetilde{X}, \widetilde{Y}, \widetilde{\phi}, \widetilde{c}, \widetilde{c}^{\vee}, \widetilde{\tau}\right)$ is the degeneration data associated with $(\widetilde{A}, \widetilde{\lambda}, \widetilde{i})$. Then $\left(\widetilde{B}, \lambda_{\widetilde{B}}, i_{\widetilde{B}}, \widetilde{X}, \widetilde{Y}, \widetilde{\phi}, \widetilde{c}, \widetilde{c}^{\vee}\right)$ (with $\widetilde{\tau}$ omitted) is isomorphic to $\left(B, \lambda_{B}, i_{B}, X, Y, \phi, c, c^{\vee}\right) \underset{R}{\otimes} \widetilde{R}$.

The two additional conditions in Theorem 3.1 mean that only the period $\widetilde{\tau}$ is being elevated. All other data are essentially intact.

The relations among the rings $R^{\diamond}, K^{\diamond}, k^{\diamond}, R, K, k, \widetilde{R}, \widetilde{K}$, and $\widetilde{k}$ can be summarized by the following commutative diagram (of their spectra):

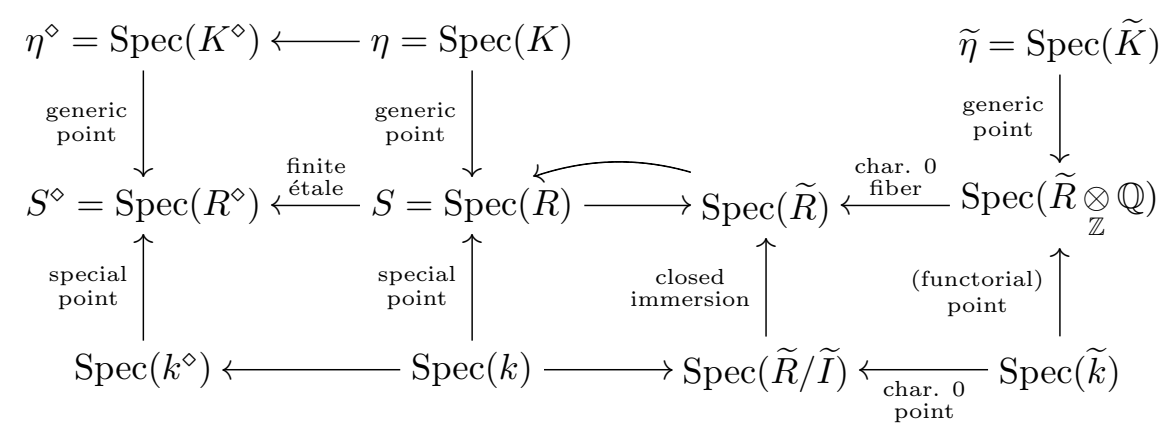

The curly arrow from $\operatorname{Spec}(\widetilde{R})$ to $S=\operatorname{Spec}(R)$ in this diagram is not required in the definition of an elevator (see Definition 2.3), but will be a byproduct of the construction.

The proof of Theorem 3.1 will be carried out in subsequent subsections. Our main references will be $[4,10]$. Our formulations follow mainly [10, Section 6.2], but our approach follows [4, Ch. IV] very closely. The main new step is the trick in the beginning of Section 3.4, which allows us to work without worrying about bad reductions.

3.1. Choice of $R$ and degeneration data. Let $R^{\diamond} \rightarrow R$ be a finite étale morphism of complete discrete valuation rings such that the pullbacks of the torus parts of $A^{\diamond}$ and $A^{\diamond \vee}$ are split over $R$. Let $S:=\operatorname{Spec}(R), K:=\operatorname{Frac}(R), \eta:=\operatorname{Spec}(K)$ the generic point of $S$, and $k$ the residue field of $R$.

The degenerating family $\left(A_{S}^{\diamond}, \lambda_{S}^{\diamond}, i_{S}^{\diamond}\right)$ of type $(\mathrm{PE}, \mathcal{O})$ over $S$ defines an object in $\operatorname{DEG}_{\mathrm{PE}, \mathcal{O}}^{\text {split }}(R)$, with degeneration data $\left(B, \lambda_{B}, i_{B}, X, Y, \phi, c, c^{\vee}, \tau\right)$ in $\operatorname{DD}_{\mathrm{PE}, \mathcal{O}}^{\mathrm{split}}(R)$.

3.2. Chart for the datum $\tau$ without positivity condition. Let $\mathbf{S}$ denote the finitely generated abelian group

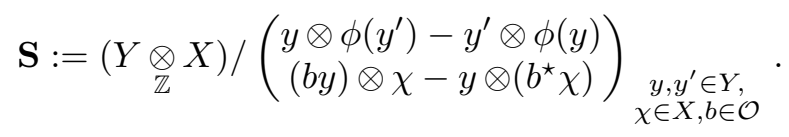


Let $\mathbf{S}_{\text {free }}$ denote the free quotient of $\mathbf{S}$, namely the quotient of $\mathbf{S}$ by its torsion subgroup $\mathbf{S}_{\text {tor }}$. Let $E:=\underline{\operatorname{Hom}}\left(\mathbf{S}, \mathbf{G}_{\mathrm{m}}\right), E_{\text {tor }}:=\underline{\operatorname{Hom}}\left(\mathbf{S}_{\text {tor }}, \mathbf{G}_{\mathrm{m}}\right)$, and $E_{\text {free }}:=\underline{\operatorname{Hom}}\left(\mathbf{S}_{\text {free }}, \mathbf{G}_{\mathrm{m}}\right)$ denote the group schemes of multiplicative type of finite type over $\operatorname{Spec}(\mathbb{Z})$. Then $E_{\text {free }}$ is a split torus, and $E_{\text {tor }}$ is finite and of multiplicative type.

For each $y \in Y$ and each $\chi \in X$, the pullback $\left(c^{\vee}(y), c(\chi)\right)^{*} \mathcal{P}_{B}$ is an invertible sheaf over $S$. The (functorial) biextension structure of $\mathcal{P}_{B}$, the symmetry of $\left(\operatorname{Id}_{B} \times \lambda_{B}\right)^{*} \mathcal{P}_{B}$, and the compatibility $c \phi=\lambda_{B} c^{\vee}$, allow us to associate a well-defined invertible sheaf $\Psi(\ell)$ with each $\ell \in \mathbf{S}$, satisfying the following properties:

(1) If $\ell=[y \otimes \chi]$, the image of $y \otimes \chi \in Y \underset{\mathbb{Z}}{\otimes} X$ under the canonical morphism $Y \underset{\mathbb{Z}}{\otimes} X \rightarrow \mathbf{S}$, then there is a canonical isomorphism $\Psi(\ell) \cong\left(c^{\vee}(y), c(\chi)\right)^{*} \mathcal{P}_{B}$.

(2) For $\ell, \ell^{\prime} \in \mathbf{S}$, we have a canonical isomorphism $\Delta_{\ell, \ell^{\prime}}^{*}: \Psi(\ell) \underset{\mathscr{O}_{S}}{\otimes} \Psi\left(\ell^{\prime}\right) \cong \Psi\left(\ell+\ell^{\prime}\right)$ of invertible sheaves over $S$.

(3) The collection of isomorphisms $\Delta_{\ell, \ell^{\prime}}^{*}$ satisfy necessary conditions making the $\mathscr{O}_{S}$-module $\underset{\ell \in \mathbf{S}}{\oplus} \Psi(\ell)$ an $\mathscr{O}_{S}$-algebra, and so that

$$
\Xi:=\underline{\operatorname{Spec}}_{\mathscr{O}_{S}}\left(\bigoplus_{\ell \in \mathbf{S}} \Psi(\ell)\right)
$$

has a canonical structure of an $E$-torsor over $S$.

(4) The same isomorphisms $\Delta_{\ell, \ell^{\prime}}^{*}$ for $\ell, \ell^{\prime} \in \mathbf{S}_{\text {tor }}$ defines similarly an $E_{\text {tor-torsor }}$

$$
\Xi_{\text {tor }}:=\underline{\operatorname{Spec}}_{\mathscr{O}_{S}}\left(\underset{\ell \in \mathbf{S}_{\text {tor }}}{\oplus} \Psi(\ell)\right)
$$

over $S$, together with a canonical (surjective) morphism $\Xi \rightarrow \Xi_{\text {tor }}$ having the

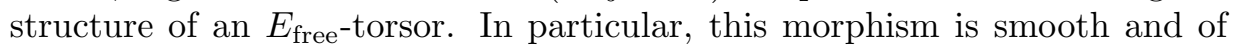
finite type.

By construction, the scheme $\Xi \rightarrow S$ is the universal space for trivializations $\mathbf{1}_{Y \times X} \stackrel{\sim}{\rightarrow}\left(c^{\vee} \times c\right)^{*} \mathcal{P}_{B}^{\otimes-1}$ of biextensions with symmetric pullbacks under $\operatorname{Id}_{Y} \times \phi$ : $Y \times Y \rightarrow Y \times X$, and with compatibility with $\mathcal{O}$-actions (but without the positivity condition). Let $\breve{\tau}$ be the universal object over $\Xi$. Then the universal property of $\Xi \rightarrow S$ determines a canonical morphism

$$
\eta \rightarrow \Xi
$$

lifting the canonical morphism $\eta \rightarrow S$, such that the trivialization $\tau: \mathbf{1}_{Y \times X, \eta} \stackrel{\sim}{\rightarrow}$ $\left(c^{\vee} \times c\right)^{*} \mathcal{P}_{B, \eta}^{\otimes-1}$ over $\eta$ is the pullback of the universal object $\breve{\tau}$ over $\Xi$.

3.3. Chart for the datum $\tau$ with positivity condition. Let $\mathbf{S}^{\vee}:=\operatorname{Hom}_{\mathbb{Z}}(\mathbf{S}, \mathbb{Z})$ and $\mathbf{S}_{\mathbb{R}}^{\vee}:=\mathbf{S}^{\vee} \underset{\mathbb{Z}}{\otimes \mathbb{R}} \cong \operatorname{Hom}_{\mathbb{Z}}(\mathbf{S}, \mathbb{R})$. By definition of $\mathbf{S}$, the $\mathbb{R}$-vector space $\mathbf{S}_{\mathbb{R}}^{\vee}$ is isomorphic to the space of symmetric pairings $(\cdot, \cdot):(Y \underset{\mathbb{Z}}{\otimes} \mathbb{R}) \times(Y \underset{\mathbb{Z}}{\otimes}) \rightarrow \mathbb{R}$ satisfying $(b x, y)=\left(x, b^{\star} y\right)$ for any $x, y \in Y \underset{\mathbb{Z}}{\otimes} \mathbb{R}$ and $b \in \mathcal{O}$. Then we define an element in $\mathbf{S}_{\mathbb{R}}^{\vee}$ to be positive definite if the associated pairing is.

A cone in $\mathbf{S}_{\mathbb{R}}^{\vee}$ is a subset stable under the natural multiplication action of the group $\mathbb{R}_{>0}$. A rational polyhedral cone in $\mathbf{S}_{\mathbb{R}}^{\vee}$ is a cone of the form $\sigma=\mathbb{R}_{>0} v_{1}+\ldots+\mathbb{R}_{>0} v_{n}$ with $v_{1}, \ldots, v_{n} \in \mathbf{S}_{\mathbb{Q}}^{\vee}=\mathbf{S}^{\vee} \underset{\mathbb{Z}}{\otimes} \mathbb{Q}$. We say that $\sigma$ is nondegenerate if its closure does not contain any nonzero $\mathbb{R}$-vector subspace. We say a nondegenerate $\sigma$ is smooth if 
the $v_{1}, \ldots, v_{n}$ can be chosen to be part of a $\mathbb{Z}$-basis of $\mathbf{S}^{\vee}$. The canonical pairing $\langle\cdot, \cdot\rangle_{\text {can. }}: \mathbf{S} \times \mathbf{S}^{\vee} \rightarrow \mathbb{Z}$ defines by extension of scalar a canonical pairing $\langle\cdot, \cdot\rangle_{\text {can. }}$ : $\mathbf{S} \times \mathbf{S}_{\mathbb{R}}^{\vee} \rightarrow \mathbb{R}$. For any rational polyhedral cone $\sigma$ in $\mathbf{S}_{\mathbb{R}}^{\vee}$, we define its dual in $\mathbf{S}$ to be the semi-group (with unit 0)

$$
\sigma^{\vee}:=\left\{x \in \mathbf{S}:\langle x, y\rangle_{\text {can. }} \geq 0, \forall y \in \sigma\right\},
$$

and we define $\sigma_{0}^{\vee}$ to be the semi-subgroup (without unit 0 ) of $\sigma^{\vee}$ defined by

$$
\sigma_{0}^{\vee}:=\left\{x \in \mathbf{S}:\langle x, y\rangle_{\text {can. }}>0, \forall y \in \sigma\right\} .
$$

Note that $\sigma^{\vee}$ always contains $\mathbf{S}_{\text {tor }}$.

For each nondegenerate rational polyhedral cone $\sigma$ in $\mathbf{S}_{\mathbb{R}}^{\vee}$, we define the affine toroidal embedding of $\Xi$ along $\sigma$ to be

$$
\Xi(\sigma):=\underline{\operatorname{Spec}}_{\mathscr{O}_{S}}\left(\bigoplus_{\ell \in \sigma^{\vee}} \Psi(\ell)\right) .
$$

Here the $\mathscr{O}_{S}$-algebra structure of $\underset{\ell \in \sigma^{\vee}}{\oplus} \Psi(\ell)$ is induced by the same morphisms $\Delta_{\ell, \ell^{\prime}}^{*}$ used in the definition $\Xi$. By construction, we have an open embedding $\Xi \hookrightarrow \Xi(\sigma)$ extending the action of $E$. Since $\sigma^{\vee}$ always contains $\mathbf{S}_{\text {tor }}$, there is a canonical surjection $\Xi(\sigma) \rightarrow \Xi_{\text {tor }}$. Then the usual theory (as in [8]) of toroidal embeddings for the torus $\mathbf{S}_{\text {free }}$ implies that the morphism $\Xi(\sigma) \rightarrow \Xi_{\text {tor }}$ is smooth if $\sigma$ is smooth.

The scheme $\Xi(\sigma)$ has a natural closed subscheme $\Xi_{\sigma}$ (often not having the same underlying topological space as the complement $\Xi(\sigma)-\Xi)$, called the $\sigma$-stratum, defined by the $\mathscr{O}_{S^{-}}$-sheaf of ideals $\underset{\ell \in \sigma_{0}^{\vee}}{\oplus} \Psi(\ell)$ in $\underset{\ell \in \sigma^{\vee}}{\oplus} \Psi(\ell)$, and is mapped to itself under the action of $E$ on $\Xi(\sigma)$. Let us define a subgroup $\sigma^{\perp}$ of $\mathbf{S}$ by

$$
\sigma^{\perp}:=\left\{x \in \mathbf{S}:\langle x, y\rangle_{\text {can. }}=0, \forall y \in \sigma\right\} .
$$

Then $E_{\sigma}:=\underline{\operatorname{Hom}}\left(\sigma^{\perp}, \mathbf{G}_{\mathrm{m}}\right)$ is a quotient group of $E$. The induced action of $E$ on $\Xi_{\sigma}$ factors through $E_{\sigma}$, and makes $\Xi_{\sigma} \rightarrow S$ a torsor under $E_{\sigma}$. Since $\sigma^{\perp}$ contains $\mathbf{S}_{\text {tor }}$, its torsion subgroup is exactly $\mathbf{S}_{\text {tor }}$. Then the canonical morphism $\Xi_{\sigma} \rightarrow \Xi_{\text {tor }}$ is surjective, and it is smooth because it is a torsor under the split torus $E_{\sigma \text {,free }}$ with character group $\sigma_{\text {free }}^{\perp}:=\sigma^{\perp} / \mathbf{S}_{\text {tor }}$.

Let $\mathbf{P}^{+}$be the cone in $\mathbf{S}_{\mathbb{R}}^{\vee}$ corresponding to positive-definite pairings. Let $v$ denote the discrete valuation of $K$ with valuation ring $R$. For each $y \in Y$ and $\chi \in X$, let $I_{y, \chi}$ be determined by the trivialization $\tau: \mathbf{1}_{Y \times X, \eta} \stackrel{\sim}{\rightarrow}\left(c^{\vee} \times c\right)^{*} \mathcal{P}_{B, \eta}^{\otimes-1}$ as in Section 2.2. Then the positivity of $\tau$ means $v\left(I_{y, \phi(y)}\right)>0$ for any $y \neq 0$, and the association $\left(y, y^{\prime}\right) \mapsto v\left(I_{y, \phi\left(y^{\prime}\right)}\right) \in \mathbb{Z}$ defines by extension of scalar a positive-definite pairing $(Y \underset{\mathbb{Z}}{\otimes} \mathbb{R}) \times(Y \underset{\mathbb{Z}}{\otimes} \mathbb{R}) \rightarrow \mathbb{R}$, corresponding to an element $b_{\tau}$ in $\mathbf{P}^{+}$.

Let us take any smooth nondegenerate rational polyhedral cone $\sigma$ in $\mathbf{P}^{+}$that contains $b_{\tau}$. Define the affine toroidal embedding $\Xi \hookrightarrow \Xi(\sigma)$ and the closed $\sigma$-stratum $\Xi_{\sigma}$ of $\Xi(\sigma)$ over $S$ as explained above. Then both $\Xi(\sigma)$ and $\Xi_{\sigma}$ are smooth over $\Xi_{\text {tor }}$. Since $\sigma$ contains $b_{\tau}$, the morphism (3.2) extends to a morphism

$$
S=\operatorname{Spec}(R) \rightarrow \Xi(\sigma)
$$

mapping the special point $\operatorname{Spec}(k)$ of $S$ to $\Xi_{\sigma}$. 
Let us summarize the construction so far in the following commutative diagram:

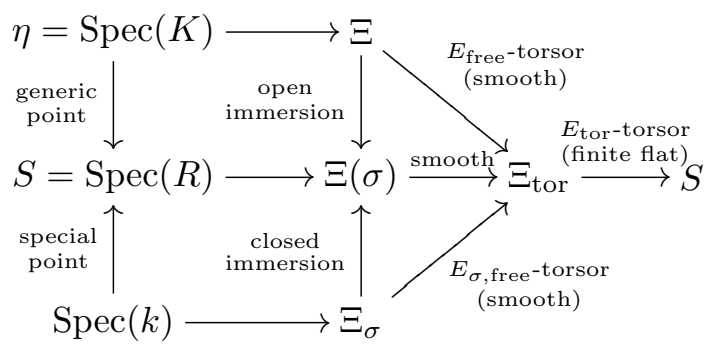

The composition of the arrows in the middle row is the identity morphism $\operatorname{Id}_{S}: S \rightarrow S$. (However, $\Xi$ is not a scheme over $\eta$, and $\Xi_{\sigma}$ is not a scheme over $\operatorname{Spec}(k)$. It is important that both $\Xi$ and $\Xi_{\sigma}$ are schemes over $S$.)

3.4. Choice of the elevator. The morphism $S \rightarrow \Xi(\sigma) \rightarrow \Xi_{\text {tor }}$ induced by (3.3) gives in particular a section of the structural morphism $\Xi_{\text {tor }} \rightarrow S$. Let us denote by $\Xi^{\circ}, \Xi^{\circ}(\sigma)$, and $\Xi_{\sigma}^{\circ}$ the pullbacks of the smooth schemes $\Xi, \Xi(\sigma)$, and $\Xi_{\sigma}$ over $\Xi_{\text {tor }}$ along this section $S \rightarrow \Xi_{\text {tor }}$. By abuse of notation, let us denote the pullback of the universal object $\breve{\tau}$ over $\Xi$ to $\Xi^{\circ}$ by the same notation $\breve{\tau}$. Then the morphism (3.3) induces a morphism

$$
S=\operatorname{Spec}(R) \rightarrow \Xi^{\circ}(\sigma)
$$

mapping $\eta=\operatorname{Spec}(K)$ to $\Xi^{\circ}$ (resp. $\operatorname{Spec}(k)$ to $\left.\Xi_{\sigma}^{\circ}\right)$, such that the trivialization $\tau$ : $\mathbf{1}_{Y \times X, \eta} \stackrel{\sim}{\rightarrow}\left(c^{\vee} \times c\right)^{*} \mathcal{P}_{B, \eta}^{\otimes-1}$ over $\eta$ is the pullback of $\breve{\tau}$ over $\Xi^{\circ}$.

Let $\widetilde{R}^{\text {pre }}$ be the local ring of $\Xi^{\circ}(\sigma)$ at the image of $\operatorname{Spec}(k) \rightarrow \Xi^{\circ}(\sigma)$. Let $\widetilde{I}^{\text {pre }}$ be the ideal of definition of the $\sigma$-stratum of $\operatorname{Spec}\left(\widetilde{R}^{\text {pre }}\right)$ induced by the $\sigma$-stratum $\Xi_{\sigma}^{\circ}$ of $\Xi^{\circ}(\sigma)$. (The ideal $\widetilde{I}^{\text {pre }}$ is not supposed to be the same as the maximal ideal defining the image of $\operatorname{Spec}(k)$.) Let $\widetilde{R}$ be the completion of $\widetilde{R}^{\text {pre }}$ with respect to $\widetilde{I}^{\text {pre }}$, and let $\widetilde{I}$ be the induced ideal of definition. Since $\Xi^{\circ}(\sigma)$ is smooth over $\operatorname{Spec}(R)$, the ring $\widetilde{R}$ is a noetherian normal domain. Then the data of $\widetilde{R}$ and $\widetilde{I}$ allow us to talk about the categories $\operatorname{DEG}_{\mathrm{PE}, \mathcal{O}}^{\text {split }}(\widetilde{R}, \widetilde{I})$ and $\operatorname{DD}_{\mathrm{PE}, \mathcal{O}}^{\text {split }}(\widetilde{R}, \widetilde{I})$. (See Section 2.2.) Let $\widetilde{K}:=\operatorname{Frac}(\widetilde{R})$ and $\widetilde{\eta}:=\operatorname{Spec}(\widetilde{K})$.

Then we have an updated commutative diagram:

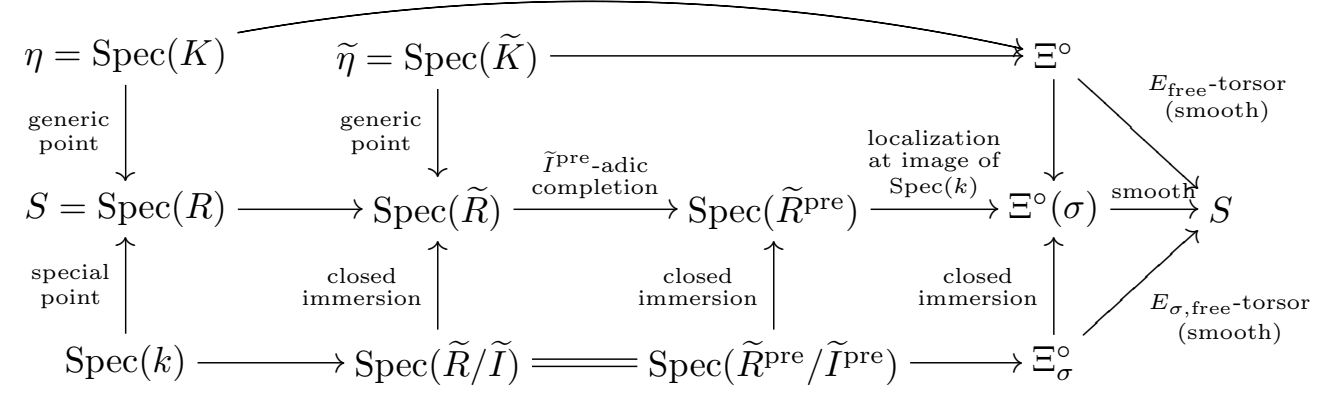

Let $\left(\widetilde{B}, \lambda_{\widetilde{B}}, i_{\widetilde{B}}, X, Y, \phi, \widetilde{c}, \widetilde{c}^{\vee}\right)$ be the pullback of $\left(B, \lambda_{B}, i_{B}, X, Y, \phi, c, c^{\vee}\right)$ to $\widetilde{R}$, and let $\widetilde{\tau}: \mathbf{1}_{Y \times X, \widetilde{\eta}} \stackrel{\sim}{\rightarrow}\left(c^{\vee} \times c\right)^{*} \mathcal{P}_{B, \tilde{\eta}}^{\otimes-1}$ be the pullback of $\breve{\tau}$ to $\widetilde{\eta}$. Then we obtain 
a tautological tuple $\left(\widetilde{B}, \lambda_{\widetilde{B}}, i_{\widetilde{B}}, X, Y, \phi, \widetilde{c}, \widetilde{c}^{\vee}, \widetilde{\tau}\right)$ over $\widetilde{R}$, which defines an object in $\mathrm{DD}_{\mathrm{PE}, \mathcal{O}}^{\text {split }}(\widetilde{R}, \widetilde{I})$. Accordingly, Mumford's construction $\mathrm{M}_{\mathrm{PE}, \mathcal{O}}^{\text {split }}(\widetilde{R}, \widetilde{I})$ gives an object $(\widetilde{A}, \widetilde{\lambda}, \widetilde{i})$ of $\operatorname{DEG}_{\mathrm{PE}, \mathcal{O}}^{\text {split }}(\widetilde{R}, \widetilde{I})$. By functoriality of Mumford's construction, the pullback of $(\widetilde{A}, \widetilde{\lambda}, \widetilde{i}) \rightarrow \operatorname{Spec}(\widetilde{R})$ along the morphism $\operatorname{Spec}(R) \rightarrow \operatorname{Spec}(\widetilde{R})$ induced by (3.4) is isomorphic to $\left(A^{\diamond}, \lambda^{\diamond}, i^{\diamond}\right) \underset{R^{\diamond}}{\otimes} R \rightarrow \operatorname{Spec}(R)$.

Since $\Xi_{\sigma}^{\circ}$ is smooth over $S=\operatorname{Spec}(R)$, the $\sigma$-stratum of $\operatorname{Spec}(\widetilde{R})$ has at least one (functorial) point $\operatorname{Spec}(\widetilde{k})$ in characteristic zero. Since the character groups of the torus parts of $\widetilde{A}$ are constant and equal to $X$ for points on the $\sigma$-stratum of $\operatorname{Spec}(\widetilde{R})$, the dimensions of the torus parts of $\widetilde{A}_{\widetilde{k}}$ and $A_{k}$ are the same.

Thus, the data of $\operatorname{Spec}(R) \rightarrow \operatorname{Spec}\left(R^{\diamond}\right), \operatorname{Spec}(R) \rightarrow \operatorname{Spec}(\widetilde{R}), \operatorname{Spec}(\widetilde{k}) \rightarrow \operatorname{Spec}(\widetilde{R})$, and $(\widetilde{A}, \widetilde{\lambda}, \widetilde{i}) \rightarrow \operatorname{Spec}(\widetilde{R})$ define an elevator for $\left(A^{\diamond}, \lambda^{\diamond}, i^{\diamond}\right) \rightarrow \operatorname{Spec}\left(R^{\diamond}\right)$ as in Definition 2.3. This completes the proof of Theorem 3.1.

\section{Upper bounds for maximal ranks of degenerations}

\subsection{Application of existence of elevators.}

Theorem 4.1. With the setting as in Theorem 1.2, we have the inequality $r_{p} \leq r_{0}$ for every $p \geq 0$.

Proof. Suppose that there is a degeneration $A^{\diamond}$ of rank $r$ over some discrete valuation ring $R^{\diamond}$ based at $\mathrm{Sh}_{\mathcal{H} \text {,can }}$. The induced morphism $\operatorname{Spec}\left(K^{\diamond}\right) \rightarrow \mathrm{Sh}_{\mathcal{H} \text {,can }} \hookrightarrow \mathrm{M}_{\mathcal{H}}$ defines an object $\left(A_{1}^{\diamond}, \lambda_{1}^{\diamond}, i_{1}^{\diamond}, \alpha_{\mathcal{H}}^{\diamond}\right)$ of $\mathrm{M}_{\mathcal{H}}\left(\operatorname{Spec}\left(K^{\diamond}\right)\right)$, such that $A_{1}^{\diamond}$ is isomorphic to the pullback of $A^{\diamond}$ to $K^{\diamond}$. By a result of Raynaud [4, Ch. I, 2.8] (or [10, Proposition 3.3.1.7]), the additional structures $\left(\lambda_{1}^{\diamond}, i_{1}^{\diamond}\right)$ of $A_{1}^{\diamond}$ extend uniquely to structures $\left(\lambda^{\diamond}, i^{\diamond}\right)$ of $A^{\diamond}$ over $R^{\diamond}$. Then we have a degenerating family $\left(A^{\diamond}, \lambda^{\diamond}, i^{\diamond}\right) \rightarrow \operatorname{Spec}\left(R^{\diamond}\right)$ of type $(\mathrm{PE}, \mathcal{O})$ extending $\left(A_{1}^{\diamond}, \lambda_{1}^{\diamond}, i_{1}^{\diamond}\right)$. Moreover, Theorem 3.1 guarantees the existence of a degenerating family $(\widetilde{A}, \widetilde{\lambda}, \widetilde{i}) \rightarrow \operatorname{Spec}(\widetilde{R})$ such that the pullback of $\widetilde{A}$ to $\operatorname{Spec}(\widetilde{R} \underset{\mathbb{Q}}{\mathbb{Q}})$ has a fiber of rank $r$.

Let $S_{1}$ be the maximal dense subscheme of $\operatorname{Spec}(\widetilde{R} \otimes \mathbb{Q})$ over which $\widetilde{A}$ is an abelian scheme. Let $K^{\text {sep }}$ denote a separable closure of $K$. Then there exists an affine integral scheme $S_{1}^{\prime}$ finite étale over $S_{1}$, together with a morphism $\operatorname{Spec}\left(K^{\text {sep }}\right) \rightarrow S_{1}^{\prime}$ lifting $\operatorname{Spec}(K) \rightarrow S_{1}$, such that $\left(\widetilde{A}_{S_{1}^{\prime}}, \widetilde{\lambda}_{S_{1}^{\prime}}, \widetilde{i}_{S_{1}^{\prime}}\right)$ satisfies the Lie algebra condition given by $\left(L \underset{\mathbb{Z}}{\otimes} \mathbb{R},\langle\cdot, \cdot\rangle, h_{0}\right)$ and is equipped with a level $\mathcal{H}$-structure $\widetilde{\alpha}_{\mathcal{H}}$ of type $(L \underset{\mathbb{Z}}{\otimes} \hat{\mathbb{Z}},\langle\cdot, \cdot\rangle)$, and such that the pullbacks of $\left(\widetilde{A}_{S_{1}^{\prime}}, \widetilde{\lambda}_{S_{1}^{\prime}}, \widetilde{i}_{S_{1}^{\prime}}, \widetilde{\alpha}_{\mathcal{H}}\right) \rightarrow S_{1}^{\prime}$ and $\left(A_{1}^{\diamond}, \lambda_{1}^{\diamond}, i_{1}^{\diamond}, \alpha_{\mathcal{H}}^{\diamond}\right) \rightarrow$ $\operatorname{Spec}\left(K^{\diamond}\right)$ to $\operatorname{Spec}\left(K^{\mathrm{sep}}\right)$ are isomorphic to each other. (See [10, Definition 1.3.4.2, Lemma 1.2.5.13, and Corollary 1.3.6.7].) Let $S_{1}^{\prime} \rightarrow \mathrm{M}_{\mathcal{H}}$ be the morphism determined by $\left(\widetilde{A}_{S_{1}^{\prime}}, \widetilde{\lambda}_{S_{1}^{\prime}}, \widetilde{i}_{S_{1}^{\prime}}, \widetilde{\alpha}_{\mathcal{H}}\right)$ by the universal property of $\mathrm{M}_{\mathcal{H}}$. By construction, the two compositions of morphisms $\operatorname{Spec}\left(K^{\mathrm{sep}}\right) \rightarrow S_{1}^{\prime} \rightarrow \mathrm{M}_{\mathcal{H}}$ and $\operatorname{Spec}\left(K^{\mathrm{sep}}\right) \rightarrow \operatorname{Spec}(K) \rightarrow$ $\operatorname{Spec}\left(K^{\diamond}\right) \rightarrow \mathrm{Sh}_{\mathcal{H}, \text { can }} \hookrightarrow \mathrm{M}_{\mathcal{H}}$ define the same point. Since $S_{1}^{\prime}$ is connected, the canonical morphism $S_{1}^{\prime} \rightarrow \mathrm{M}_{\mathcal{H}}$ factors through $S_{1}^{\prime} \rightarrow \mathrm{Sh}_{\mathcal{H} \text {,can }} \hookrightarrow \mathrm{M}_{\mathcal{H}}$.

Let $R^{\Delta}$ be a discrete valuation ring, with a morphism $\operatorname{Spec}\left(R^{\Delta}\right) \rightarrow \operatorname{Spec}(\widetilde{R} \underset{\mathbb{Z}}{\otimes})$ whose restriction to the generic point factors through $S_{1}^{\prime} \rightarrow \operatorname{Spec}(\widetilde{R} \underset{\mathbb{Q}}{\otimes})$ and whose 
restriction to the special point factors through $\operatorname{Spec}(\widetilde{k}) \rightarrow \operatorname{Spec}(\widetilde{R} \underset{\mathbb{Z}}{\otimes})$. Such an $R^{\triangle}$ exists because $\operatorname{Spec}(\widetilde{R} \underset{\mathbb{Z}}{\otimes})$ is noetherian and integral. Then the pullback of $(\widetilde{A}, \widetilde{\lambda}, \widetilde{i}) \rightarrow \operatorname{Spec}(\widetilde{R})$ to $\operatorname{Spec}\left(R^{\Delta}\right)$ is a degeneration of rank $r$ based at $\operatorname{Sh}_{\mathcal{H}, \text { can }}$, showing that $r \leq r_{0}$ as desired.

The relations among the base schemes involved in the proof of Theorem 4.1 can be summarized by the following commutative diagram (cf. the diagram following Theorem 3.1), in which the dotted arrows are induced by the solid arrows by the connectedness of $S_{1}^{\prime}$ :

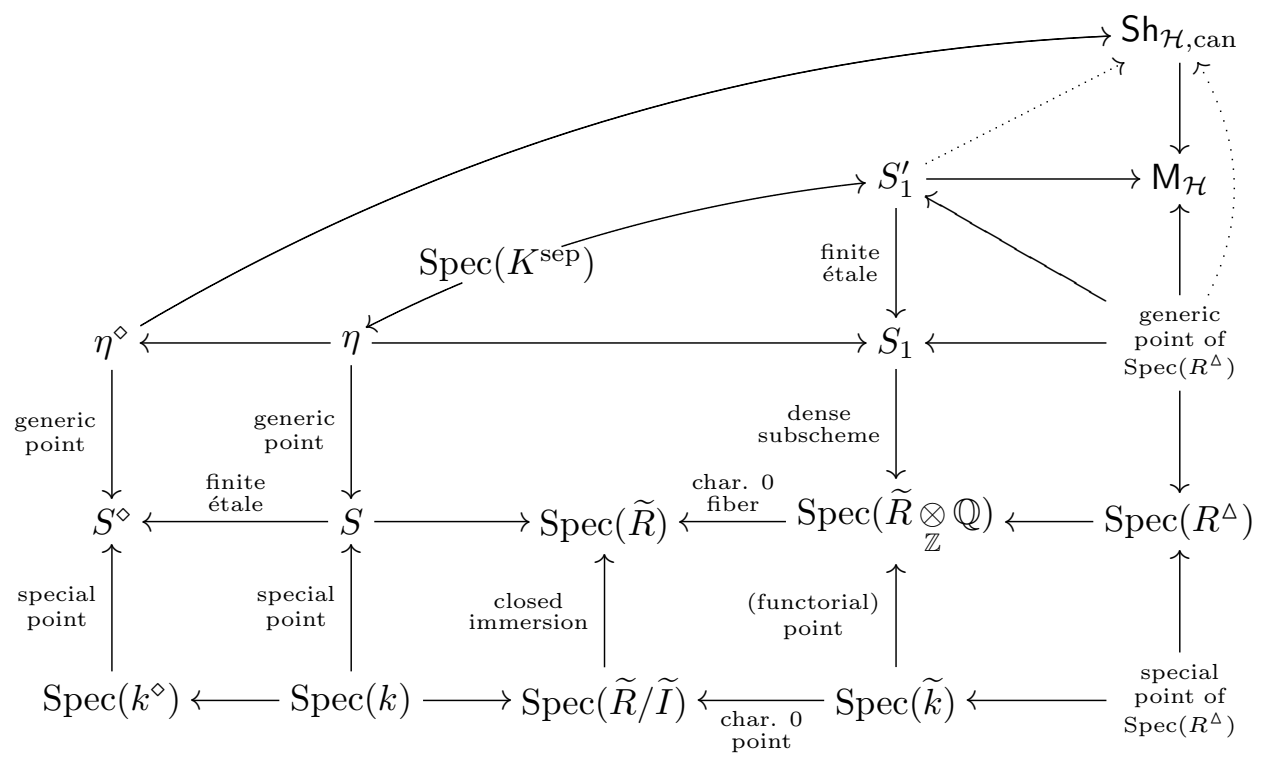

Corollary 4.2 (of Theorem 4.1). With the setting as in Theorem 1.2 , if $r_{0}=0$, then $r_{p}=0$ for every $p \geq 0$.

4.2. Morita's conjecture. Yasuo Morita's original conjecture in [12], concerning potential good reduction everywhere of abelian varieties with PEL structures, can be reformulated in our language as follows:

Conjecture 4.3 (Yasuo Morita). With the setting as above, if the analytic space $\mathrm{Sh}_{\mathcal{H}}$ is compact, then $r_{p}=0$ for every $p \geq 0$.

Remark 4.4. By [3, 11.4 and 11.6], the assumption that the analytic $\operatorname{space} \mathrm{Sh}_{\mathcal{H}}$ is compact is equivalent to the assumption that the group $G(\mathbb{Q})$ contains no unipotent element other than the identity.

Let us begin with a simple reduction step:

Lemma 4.5. The analytic space $\mathrm{Sh}_{\mathcal{H}}$ is compact if and only if the scheme $\mathrm{Sh}_{\mathcal{H} \text {,can is }}$ projective (and hence proper) over $\operatorname{Spec}\left(F_{0}\right)$.

Proof. By $[2,10.11], \mathrm{Sh}_{\mathcal{H}, \text { alg }}$ is projective over $\operatorname{Spec}(\mathbb{C})$ if $\mathrm{Sh}_{\mathcal{H}}$ is compact. By [6, IV-3, 9.1.5], $\operatorname{Sh}_{\mathcal{H}, \text { can }}$ is projective over $\operatorname{Spec}\left(F_{0}\right)$ because $\operatorname{Sh}_{\mathcal{H}, \text { alg }} \cong \operatorname{Sh}_{\mathcal{H} \text {,can }} \underset{F_{0}}{\otimes}$. 
Theorem 4.6. Conjecture 4.3 is true.

Proof. By Lemma 4.5, we see that $r_{0}=0$ if the analytic space $\mathrm{Sh}_{\mathcal{H}}$ is compact. Then Conjecture 4.3 follows from Corollary 4.2 .

Remark 4.7. Although our proof of Conjecture 4.3 might appear to be simple, we would like to mention that our approach is (as yet) the only known one that does not involve assumptions of the following kinds:

(1) The size of $L$ is small in the sense that $L \underset{\mathbb{Z}}{\otimes} \mathbb{Q}$ is (or is close to being) a simple module of $\mathcal{O} \underset{\mathbb{Z}}{\mathbb{Q}}$. (See [12] or [9, end of Section 5] for more precise statements.)

(2) For some (archimedean or non-archimedean) place $v$ of $\mathbb{Q}$, the group $G\left(\mathbb{Q}_{v}\right)$ (or some analogue using some Mumford-Tate group) contains no unipotent element other than the identity. (See $[12,14,16]$.)

Remark 4.8. Since the proof of [16] uses [4, Ch. IV, Theorem 6.7], which is in turn based on a delicate gluing argument beyond the application of the theory of degeneration data in [4, Chs. II and III], our approach is logically the simplest among the known ones on Morita's original conjecture in [12]. Nevertheless, we do not claim that our approach can be modified to tackle the stronger version of Morita's conjecture formulated using Mumford-Tate groups as in [14, 16].

Let us include a criterion of properness strengthening [10, Theorem 5.3.3.1]:

Theorem 4.9. Let $\mathcal{O}_{F_{0}}^{\prime}$ be the localization of $\mathcal{O}_{F_{0}}$ at some (possibly empty) multiplicative subset of non-units. Let $\mathrm{M}^{\prime}$ be a scheme separated of finite type over $\operatorname{Spec}\left(\mathcal{O}_{F_{0}}^{\prime}\right)$. Suppose the following conditions are satisfied:

(1) There is an open dense subscheme $\mathrm{M}^{\prime \prime}$ of $\mathrm{M}^{\prime}$ such that $\mathrm{M}^{\prime \prime} \rightarrow \operatorname{Spec}\left(\mathcal{O}_{F_{0}}^{\prime}\right)$ is smooth.

(2) There exists a morphism $f: \mathrm{M}_{F_{0}}^{\prime}:=\mathrm{M}^{\prime} \underset{\mathcal{O}_{F_{0}}^{\prime}}{\otimes} F_{0} \rightarrow \mathrm{M}_{\mathcal{H}}\left(\right.$ over $\left.\operatorname{Spec}\left(F_{0}\right)\right)$ such that, for any complete discrete valuation ring $R^{\diamond}$ with fraction field $K^{\diamond}$ of characteristic zero, and with algebraically closed residue field, a morphism $\xi_{1}: \operatorname{Spec}\left(K^{\diamond}\right) \rightarrow \mathrm{M}_{F_{0}}^{\prime}$ defining an object $\left(A_{1}^{\diamond}, \lambda_{1}^{\diamond}, i_{1}^{\diamond}, \alpha_{\mathcal{H}}^{\diamond}\right)$ of $\mathrm{M}_{\mathcal{H}}\left(\operatorname{Spec}\left(K^{\diamond}\right)\right)$ by composition with $f$ extends to a morphism $\xi: \operatorname{Spec}\left(R^{\diamond}\right) \rightarrow \mathrm{M}^{\prime}$ whenever the abelian scheme $A_{1}^{\diamond}$ extends to an abelian scheme over $\operatorname{Spec}\left(R^{\diamond}\right)$.

(3) The morphism $f$ in (2) factors through some open and closed subscheme $\mathrm{M}^{\circ} \hookrightarrow \mathrm{M}_{\mathcal{H}}$.

(4) The scheme $\mathrm{M}^{\circ}$ is proper over $\operatorname{Spec}\left(F_{0}\right)$.

Then $\mathrm{M}^{\prime} \rightarrow \operatorname{Spec}\left(\mathcal{O}_{F_{0}}^{\prime}\right)$ is proper.

Proof. To show that $\mathrm{M}^{\prime} \rightarrow \operatorname{Spec}\left(\mathcal{O}_{F_{0}}^{\prime}\right)$ is proper, we need to verify the valuative criterion for it. By (1), it suffices to show that, for any $\operatorname{Spec}\left(R^{\diamond}\right) \rightarrow \operatorname{Spec}\left(\mathcal{O}_{F_{0}}^{\prime}\right)$ where $R^{\diamond}$ is a complete discrete valuation ring $R^{\diamond}$ with fraction field $K^{\diamond}$ (of characteristic zero) and with algebraically closed residue field $k^{\diamond}$, any morphism $\xi_{1}: \operatorname{Spec}\left(K^{\diamond}\right) \rightarrow$ $\mathrm{M}_{F_{0}}^{\prime}$ extends to a morphism $\xi: \operatorname{Spec}\left(R^{\diamond}\right) \rightarrow \mathrm{M}^{\prime}$.

By composition with $f$ in $(2)$, the morphism $\xi_{1}: \operatorname{Spec}\left(K^{\diamond}\right) \rightarrow \mathrm{M}_{F_{0}}^{\prime}$ induces a morphism $f \circ \xi_{1}: \operatorname{Spec}\left(K^{\diamond}\right) \rightarrow \mathrm{M}_{\mathcal{H}}$ defining an object $\left(A_{1}^{\diamond}, \lambda_{1}^{\diamond}, i_{1}^{\diamond}, \alpha_{\mathcal{H}}^{\diamond}\right)$ of $\mathrm{M}_{\mathcal{H}}\left(\operatorname{Spec}\left(K^{\diamond}\right)\right)$. 
Then, as in the first paragraph of the proof of Theorem 4.1, we have a degenerating family $\left(A^{\diamond}, \lambda^{\diamond}, i^{\diamond}\right)$ of type $(\mathrm{PE}, \mathcal{O})$ over $\operatorname{Spec}\left(R^{\diamond}\right)$ extending $\left(A_{1}^{\diamond}, \lambda_{1}^{\diamond}, i_{1}^{\diamond}\right)$.

By Theorem 3.1, we obtain a degenerating family $(\widetilde{A}, \widetilde{\lambda}, \widetilde{i})$ of type $(\mathrm{PE}, \mathcal{O})$ over $\widetilde{R}$, together with morphisms $\operatorname{Spec}(R) \rightarrow \operatorname{Spec}\left(R^{\diamond}\right)$ and $\operatorname{Spec}(R) \rightarrow \operatorname{Spec}(\widetilde{R})$ satisfying the additional properties in Definition 2.3. Let $S_{1}$ be the maximal dense subscheme of $\operatorname{Spec}(\widetilde{R} \otimes \mathbb{Q})$ over which $\widetilde{A}$ is an abelian scheme, and let $K^{\text {sep }}$ denote a separable closure of $\underset{K}{K}$. As in the second paragraph of the proof of Theorem 4.1, there exists a finite étale morphism $S_{1}^{\prime} \rightarrow S_{1}$ from an integral scheme, such that $\left(\widetilde{A}_{S_{1}^{\prime}}, \widetilde{\lambda}_{S_{1}^{\prime}}, \widetilde{i}_{S_{1}^{\prime}}\right)$ is equipped with the additional structures defining a canonical morphism $S_{1}^{\prime} \rightarrow \mathrm{M}_{\mathcal{H}}$, and such that the composition of morphisms $\operatorname{Spec}\left(K^{\mathrm{sep}}\right) \rightarrow \operatorname{Spec}(K) \rightarrow \operatorname{Spec}\left(K^{\diamond}\right) \stackrel{f \circ \xi_{1}}{\rightarrow} \mathrm{M}_{\mathcal{H}}$ factors through this $S_{1}^{\prime} \rightarrow \mathrm{M}_{\mathcal{H}}$. Then $S_{1}^{\prime} \rightarrow \mathrm{M}_{\mathcal{H}}$ factors through $S_{1}^{\prime} \rightarrow \mathrm{M}^{\circ} \hookrightarrow \mathrm{M}_{\mathcal{H}}$ by (3) and the connectedness of $S_{1}^{\prime}$.

Consider an arbitrary spectrum of a discrete valuation ring with generic point above $S_{1}$ and with special point above a point of $\operatorname{Spec}(\widetilde{R} \underset{\mathbb{Q}}{\otimes})$. The pullback of $(\widetilde{A}, \widetilde{\lambda}, \widetilde{i})$ to this spectrum defines a degeneration of an object of $\mathrm{M}^{\circ} \hookrightarrow \mathrm{M}_{\mathcal{H}}$. By the usual theory of Néron models, or by a result of Raynaud [4, Ch. I, 2.7] (or [10, Proposition 3.3.1.7]), the degeneration is uniquely determined by its generic fiber. By (4), such a degeneration has to be rank zero, which means its special fiber is above a point of $S_{1}$. Hence $S_{1}$ has to be the whole $\operatorname{Spec}(\widetilde{R} \underset{\mathbb{Z}}{\otimes} \mathbb{Q})$.

Since there exists at least one point $\operatorname{Spec}(\widetilde{k}) \rightarrow \operatorname{Spec}(\widetilde{R} \underset{\mathbb{Z}}{\otimes})$ such that the torus part of $\widetilde{A}_{\widetilde{k}}$ has the same dimension as the torus part of $A_{k^{\diamond}}^{\diamond}$, we see that the torus part of $A_{k^{\diamond}}^{\diamond}$ is trivial. This shows that $A^{\diamond} \rightarrow \operatorname{Spec}\left(R^{\diamond}\right)$ is an abelian scheme, and the theorem follows from (2).

Corollary 4.10. Let $\mathrm{M}^{\circ}$ be an open and closed subscheme of $\mathrm{M}_{\mathcal{H}}$. (For example, we can take $\mathrm{M}^{\circ}$ to be the image of the canonical embedding $\mathrm{Sh}_{\mathcal{H} \text {,can }} \hookrightarrow \mathrm{M}_{\mathcal{H}}$.) Consider the natural (quasi-finite) morphism from $\mathrm{M}^{\circ}$ to a suitable coarse Siegel moduli scheme (with possibly non-principal polarizations) over $\operatorname{Spec}(\mathbb{Z})$ (constructed in [13]). Take the closure of the schematic image of this natural morphism, and take the normalization $\mathrm{M}^{\prime}$ of this closure in the "function field" of $\mathrm{M}^{\circ}$. (We allow the "function field" to be a product of fields.) If $\mathrm{M}^{\circ} \rightarrow \operatorname{Spec}\left(F_{0}\right)$ is proper, then the scheme $\mathrm{M}^{\prime}$ is projective over $\operatorname{Spec}\left(\mathcal{O}_{F_{0}}\right)$.

Proof. By construction, the conditions in Theorem 4.9 are all satisfied by $\mathrm{M}^{\prime}$, with $\mathcal{O}_{F_{0}}^{\prime}=\mathcal{O}_{F_{0}}$ (localizing at the empty set). Thus $\mathrm{M}^{\prime}$ is proper over $\operatorname{Spec}\left(\mathcal{O}_{F_{0}}\right)$ by Theorem 4.9, and hence projective over $\operatorname{Spec}\left(\mathcal{O}_{F_{0}}\right)$ by quasi-projectivity of the coarse Siegel moduli scheme over $\operatorname{Spec}(\mathbb{Z})$.

\section{Lower bounds for maximal ranks of degenerations}

Since the ranks of fibers of a semi-abelian scheme are non-deceasing under specialization (by arguments using torsion points, as in [5, IX, 2.2.1 and 2.2.3]), it is natural to expect the following result:

Theorem 5.1. With the setting as in Theorem 1.2, we have the equality $r_{0} \leq r_{p}$ for every characteristic $p \geq 0$. 
To prove Theorem 5.1, it suffices to construct a degeneration of rank $r_{0}$ over a discrete valuation ring with residue characteristic $p$ for each characteristic $p \geq 0$. Although alternative approaches might exist, we prefer to exploit an implication of the main results of [10], so that a construction similar to the one for elevators in Section 2.3 can be carried out. (However, we will only need the conclusion of [10] in characteristic zero. That is, although we will use the algebraic construction of the toroidal compactifications over $\operatorname{Spec}\left(F_{0}\right)$, we will never use their smooth integral models.)

5.1. Boundary stratum of toroidal compactifications. Let us fix a characteristic $p \geq 0$.

According to [10, Theorems 6.4.1.1 and 7.3.3.4], $\mathrm{M}_{\mathcal{H}} \rightarrow \operatorname{Spec}\left(F_{0}\right)$ admits a projective smooth toroidal compactification $\mathrm{M}_{\mathcal{H}}^{\text {tor }}=\mathrm{M}_{\mathcal{H}, \Sigma}^{\text {tor }} \rightarrow \operatorname{Spec}\left(F_{0}\right)$, subject to some combinatorial choice of $\Sigma$, with a stratification by locally closed subschemes. The universal object over $\mathrm{M}_{\mathcal{H}}$ extends (after forgetting level structures) to a degenerating family $(A, \lambda, i)$ of type $(\mathrm{PE}, \mathcal{O})$ over $\mathrm{M}_{\mathcal{H}}^{\text {tor }}$, and the restriction of $A$ to each stratum of $\mathrm{M}_{\mathcal{H}}^{\text {tor }}$ has constant (split) torus parts. Let $\mathrm{Sh}_{\mathcal{H}}^{\text {tor } \text {,an }}$ denote the schematic closure of $\mathrm{Sh}_{\mathcal{H} \text {,can }}$ in $\mathrm{M}_{\mathcal{H}}^{\text {tor }}$. Since $\mathrm{Sh}_{\mathcal{H} \text {,can }} \hookrightarrow \mathrm{M}_{\mathcal{H}}$ is open and closed, and since $\mathrm{M}_{\mathcal{H}}^{\text {tor }}$ is regular, $\mathrm{Sh}_{\mathcal{H} \text {,can }}^{\text {tor }} \hookrightarrow \mathrm{M}_{\mathcal{H}}^{\text {tor }}$ is again open and closed. The stratification of $\mathrm{M}_{\mathcal{H}}^{\text {tor }}$ thus induces a stratification of $\mathrm{Sh}_{\mathcal{H}}^{\text {tor } \text { can }}$ with similar properties. By the semi-stable reduction theorem [4, Ch. I, 2.6] (or [10, Theorem 3.3.2.4]), the properness of $\operatorname{Sh}_{\mathcal{H}, \text { can }}^{\text {tor }} \rightarrow \operatorname{Spec}\left(F_{0}\right)$ shows that the maximal rank $r_{0}$ among degenerations over equicharacteristic zero discrete valuation rings based at $\mathrm{Sh}_{\mathcal{H} \text {,can }}$ is achieved over at least one (locally closed) stratum of $\mathrm{Sh}_{\mathcal{H} \text {,can }}^{\text {tor }}$ Moreover, since ranks of fibers of a semi-abelian scheme are non-deceasing under specialization (as mentioned above), we may require that this stratum is closed (and hence proper over $\operatorname{Spec}\left(F_{0}\right)$ ). Let us pick any such (closed) stratum $\mathrm{Z}^{\circ}$ of $\mathrm{Sh}_{\mathcal{H} \text {,can }}^{\text {tor }}$, which is the intersection of $\mathrm{Sh}_{\mathcal{H} \text {,can }}^{\text {tor }}$ with a (locally closed) stratum $\mathrm{Z}$ of $\mathrm{M}_{\mathcal{H}}^{\text {tor }}$.

Proposition 5.2. There exists a degeneration $\left(A^{\circ}, \lambda^{\circ}, i^{\circ}\right)$ over a complete discrete

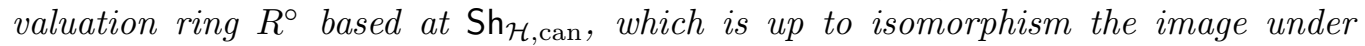
$\mathrm{M}_{\mathrm{PE}, \mathcal{O}}^{\text {split }}\left(R^{\circ}\right)$ of some tuple $\left(B^{\circ}, \lambda_{B^{\circ}}, i_{B^{\circ}}, X, Y, \phi, c^{\circ}, c^{\circ \vee}, \tau^{\circ}\right)$ in $\operatorname{DD}_{\mathrm{PE}, \mathcal{O}}^{\text {split }}\left(R^{\circ}\right)$, satisfying the following conditions:

(1) There exists a morphism $S^{\circ}:=\operatorname{Spec}\left(R^{\circ}\right) \rightarrow \mathrm{M}_{\mathcal{H}}^{\text {tor }}$, with generic point of $S^{\circ}$ mapped to $\mathrm{Sh}_{\mathcal{H} \text {,can }}$ and special point of $S^{\circ}$ mapped to $\mathrm{Z}^{\circ}$, such that $\left(A^{\circ}, \lambda^{\circ}, i^{\circ}\right)$ is isomorphic to the pullback of $(A, \lambda, i)$ under this morphism. Then $\operatorname{rk}_{\mathbb{Z}} X=$ $\mathrm{rk}_{\mathbb{Z}} Y=r_{0}$ according to the choice of $\mathrm{Z}^{\circ}$.

(2) There exists a complete discrete valuation ring $R$ of residue characteristic $p$, with fraction field $K:=\operatorname{Frac}(R)$ the residue field of $R^{\circ}$, together with a tuple $\left(B, \lambda_{B}, i_{B}, X, Y, \phi, c, c^{\vee}\right)$ forming part of an object in $\mathrm{DD}_{\mathrm{PE}, \mathcal{O}}^{\mathrm{split}}(R)$, such that

$$
\left(B^{\circ}, \lambda_{B^{\circ}}, i_{B^{\circ}}, X, Y, \phi, c^{\circ}, c^{\circ \vee}\right) \cong\left(B, \lambda_{B}, i_{B}, X, Y, \phi, c, c^{\vee}\right) \underset{R}{\otimes} R^{\circ} .
$$

Proof. According to the construction in [10, Section 6.2], Z is canonically a torustorsor over an abelian scheme $C^{\mathrm{Z}}$ over some moduli problem $\mathrm{M}_{\mathcal{H}}^{Z}$ (whose definition is analogous to that of $\mathrm{M}_{\mathcal{H}}$ ). Moreover, the formal completion of $\mathrm{M}_{\mathcal{H}}^{\text {tor }}$ along $\mathrm{Z}$ is isomorphic to a formal scheme $\mathfrak{X}^{\mathrm{Z}}$ over $C^{\mathrm{Z}}$. (To form the formal completion along a locally closed stratum, we first remove the other strata appearing in the closure 
of this stratum from the total space, and then form the formal completion of the remaining space along this stratum.) Then $\mathrm{Z}^{\circ}$ is also a torus-torsor (under the same torus) over an abelian scheme $C^{Z^{\circ}}$ over an open and closed subscheme $\mathrm{M}_{\mathcal{H}}^{Z^{\circ}}$ of $\mathrm{M}_{\mathcal{H}}^{Z}$, and the formal completion of $\mathrm{Sh}_{\mathcal{H} \text {,can }}^{\text {tor }}$ along $\mathrm{Z}^{\circ}$ is isomorphic to a formal scheme $\mathfrak{X}^{\mathrm{Z}^{\circ}}$ over $C^{\mathrm{Z}^{\circ}}$.

Let $R$ be any complete discrete valuation ring with algebraically closed residue field of characteristic $p$, with (characteristic zero) fraction field $K=\operatorname{Frac}(R)$, such that there exists a morphism $\operatorname{Spec}(K) \rightarrow Z^{\circ}$. This induces canonically a morphism $\xi: \operatorname{Spec}(K) \rightarrow C^{Z^{\circ}}$.

Let $R^{\circ}$ be any complete discrete valuation ring with fraction field $K^{\circ}$ and residue field $K$, together with a morphism from $\operatorname{Spf}\left(R^{\circ}\right)$ to the pullback of $\mathfrak{X}^{Z^{\circ}}$ under $\xi$, which defines canonically a morphism $\xi^{\circ}: \operatorname{Spec}\left(R^{\circ}\right) \rightarrow \mathrm{M}_{\mathcal{H}}^{\text {tor }}$ whose restriction to the generic point $\eta^{\circ}:=\operatorname{Spec}\left(K^{\circ}\right)$ of $\operatorname{Spec}\left(R^{\circ}\right)$ factors through $\operatorname{Sh}_{\mathcal{H} \text {,can }} \hookrightarrow \mathrm{M}_{\mathcal{H}}^{\text {tor }}$, and whose restriction to the special point $\operatorname{Spec}(K)$ of $\operatorname{Spec}\left(R^{\circ}\right)$ factors through $\operatorname{Spec}(K) \rightarrow Z^{\circ} \rightarrow$ $\mathrm{M}_{\mathcal{H}}^{\text {tor }}$.

Let $\left(A^{\circ}, \lambda^{\circ}, i^{\circ}\right)$ be the pullback of $(A, \lambda, i)$ under $\xi^{\circ}: \operatorname{Spec}\left(R^{\circ}\right) \rightarrow \mathrm{M}_{\mathcal{H}}^{\text {tor }}$. Since the torus part of the special fiber of $A^{\circ}$ is constant, $\left(A^{\circ}, \lambda^{\circ}, i^{\circ}\right)$ is up to isomorphism the image under $\mathrm{M}_{\mathrm{PE}, \mathcal{O}}^{\text {split }}\left(R^{\circ}\right)$ of some tuple $\left(B^{\circ}, \lambda_{B^{\circ}}, i_{B^{\circ}}, X, Y, \phi, c^{\circ}, c^{\circ \vee}, \tau^{\circ}\right)$ in $\operatorname{DD}_{\mathrm{PE}, \mathcal{O}}^{\text {split }}\left(R^{\circ}\right)$. Since the canonical morphism $\operatorname{Spf}\left(R^{\circ}\right) \rightarrow \mathfrak{X}^{\mathrm{Z}^{\circ}} \rightarrow C^{\mathrm{Z}^{\circ}}$ factors through the pullback of $\mathfrak{X}^{Z^{\circ}}$ under $\xi$, by the universal property of $C^{\mathrm{Z}}$, there exists a tuple $\left(B_{K}, \lambda_{B_{K}}, i_{B_{K}}, c_{K}, c_{K}^{\vee}\right)$ over $K$ such that $\left(B_{K}, \lambda_{B_{K}}, i_{B_{K}}, c_{K}, c_{K}^{\vee}\right) \otimes_{K}^{\circ} \cong$ $\left(B^{\circ}, \lambda_{B^{\circ}}, i_{B^{\circ}}, c^{\circ}, c^{\circ \vee}\right)$.

The relations among the base schemes involved in characteristic zero can be summarized in the following commutative diagram, in which "str." means structural morphisms and "cmpl." means formal completions:

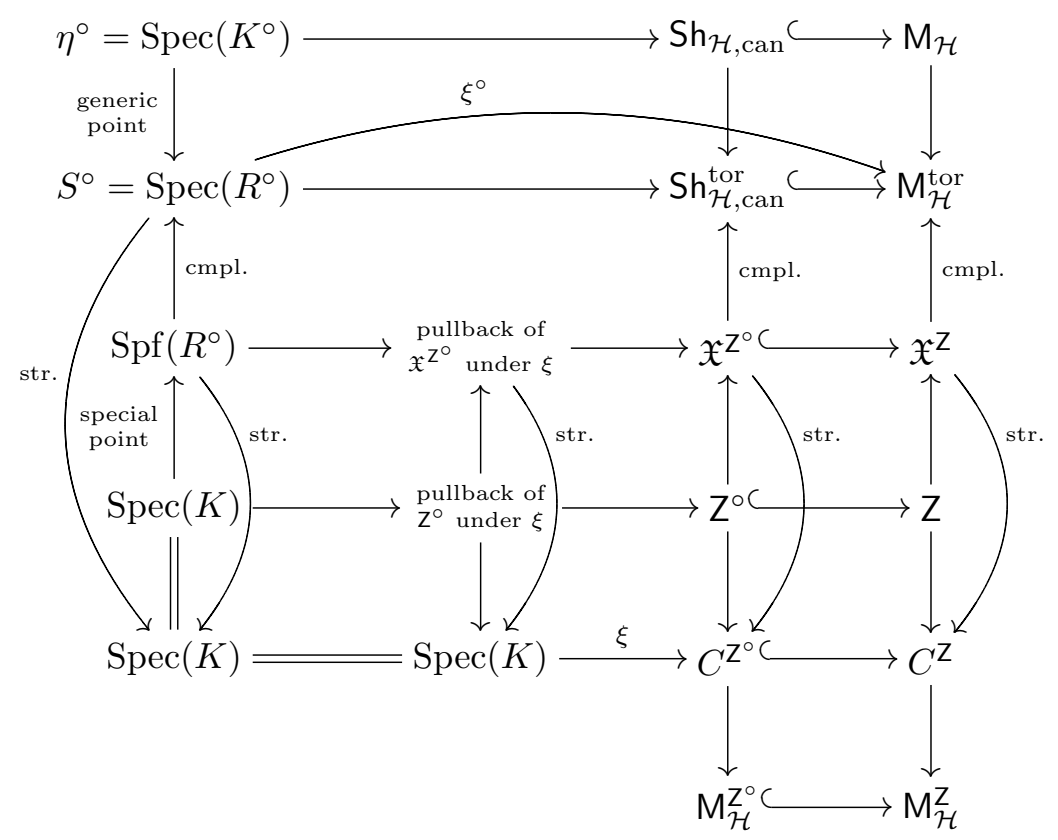


Since $\mathrm{Z}^{\circ} \rightarrow \operatorname{Spec}\left(F_{0}\right)$ is proper, $\mathrm{M}_{\mathcal{H}}^{Z^{\circ}} \rightarrow \operatorname{Spec}\left(F_{0}\right)$ is also proper. By Corollary 4.10, the abelian scheme $B_{K}$ parameterized by $\mathrm{M}_{\mathcal{H}}^{Z^{\circ}}$ has potential good reduction everywhere. In particular, it extends to an abelian scheme $B$ over $R$. By a result of Raynaud [4, Ch. I, 2.8] (or [10, Proposition 3.3.1.7]), the additional structures $\left(\lambda_{B_{K}}, i_{B_{K}}\right)$ of $B_{K}$ extend uniquely to structures $\left(\lambda_{B}, i_{B}\right)$ of $B$ over $R$. Since $X$ (resp. $Y$ ) is finitely generated, the morphism $c_{K}: X \rightarrow B_{K}^{\vee}$ (resp. $\left.c_{K}^{\vee}: Y \rightarrow B_{K}\right)$ is determined by finitely many $\operatorname{Spec}(K)$-valued points of $B_{K}^{\vee}\left(\right.$ resp. $\left.B_{K}\right)$, and each of these points extends to a unique $\operatorname{Spec}(R)$-valued point of $B^{\vee}$ (resp. $B$ ) by properness of $B^{\vee} \rightarrow \operatorname{Spec}(R)$ (resp. $B \rightarrow \operatorname{Spec}(R))$. Thus, we obtain a morphism $c: X \rightarrow B^{\vee}\left(\right.$ resp. $c^{\vee}: Y \rightarrow B$ ) extending $c_{K}$ (resp. $\left.c_{K}^{\vee}\right)$, and the tuple $\left(B, \lambda_{B}, i_{B}, X, Y, \phi, c, c^{\vee}\right)$ over $R$ satisfies (5.3), as desired.

Remark 5.4. In the proof of Proposition 5.2, we referred to Corollary 4.10 rather than the seemingly more relevant Theorem 4.6 , because $\mathrm{M}_{\mathcal{H}}^{Z^{\circ}}$ might not be the Shimura variety (analogous to $S h_{\mathcal{H} \text {,can }}$ ) attached to the data defining the moduli problem $\mathrm{M}_{\mathcal{H}}^{Z}$.

5.2. Elevators in the opposite direction. Let us keep the setting in Proposition 5.2. Let $S:=\operatorname{Spec}(R)$ and $\eta:=\operatorname{Spec}(K)$.

Using the tuple $\left(B, \lambda_{B}, i_{B}, X, Y, \phi, c, c^{\vee}\right) \rightarrow S$, let $\mathbf{S}, \mathbf{S}_{\text {free }}, \mathbf{S}_{\text {tor }}, E, E_{\text {free }}, E_{\text {tor }}, \Xi$, $\Xi_{\text {tor }}$, and $\breve{\tau}$ be constructed as in Section 3.2. Let $b_{\tau^{\circ}} \in \mathbf{P}^{+}$be defined by $\tau^{\circ}$ as in Section 3.3. Let us take any top-dimensional smooth nondegenerate rational polyhedral cone $\sigma$ in $\mathbf{P}^{+}$that contains $b_{\tau^{\circ}}$, and construct $\Xi(\sigma)$ and $\Xi_{\sigma}$ (as in Section 3.3) using the cone $\sigma$.

The degeneration data of $\left(A^{\circ}, \lambda^{\circ}, i^{\circ}\right)$ defines a canonical morphism $\operatorname{Spec}\left(R^{\circ}\right) \rightarrow$ $\Xi(\sigma)$ mapping the special point $\eta=\operatorname{Spec}(K)$ of $\operatorname{Spec}\left(R^{\circ}\right)$ to the $\sigma$-stratum $\Xi_{\sigma}$. Since $\sigma$ is top dimensional, $\sigma^{\perp}=\mathbf{S}_{\text {tor }}$ in $\mathbf{S}$. Hence, the structural morphism $\Xi_{\sigma} \rightarrow \Xi_{\text {tor }}$ is an isomorphism, and we obtain a morphism $\eta \rightarrow \Xi_{\text {tor }}$ lifting the canonical morphism $\eta \rightarrow S$. Since $\Xi_{\text {tor }} \rightarrow S$ is finite, the morphism $\eta \rightarrow \Xi_{\text {tor }}$ extends to a section of the structural morphism $\Xi_{\text {tor }} \rightarrow S$. By taking pullbacks along the section $S \rightarrow \Xi_{\sigma}$, we obtain schemes $\Xi^{\circ}, \Xi^{\circ}(\sigma)$, and $\Xi_{\sigma}^{\circ}$ smooth over $S$ as in Section 3.4, together with the universal object $\breve{\tau}$ over $\Xi^{\circ}$ by abuse of notation.

Let $\widetilde{R}^{\text {pre }}$ be the noetherian normal domain underlying the affine scheme $\Xi^{\circ}(\sigma)$ (smooth over $S=\operatorname{Spec}(R)$ ), and let $\widetilde{I}^{\text {pre }}$ be the ideal of definition of the $\sigma$-stratum $\Xi_{\sigma}^{\circ}$ of $\Xi^{\circ}(\sigma)$. Let $\widetilde{R}$ be the completion of $\widetilde{R}^{\text {pre }}$ with respect to $\widetilde{I}^{\text {pre }}$, and let $\widetilde{I}$ be the induced ideal of definition. Since $\Xi^{\circ}(\sigma)$ is smooth over $S=\operatorname{Spec}(R), \widetilde{R}^{\text {pre }}$ is excellent, and hence the ring $\widetilde{R}$ remains to be a noetherian normal domain. Then the data of $\widetilde{R}$ and $\widetilde{I}$ allow us to talk about the categories $\operatorname{DEG}_{\mathrm{PE}, \mathcal{O}}^{\text {split }}(\widetilde{R}, \widetilde{I})$ and $\operatorname{DD}_{\mathrm{PE}, \mathcal{O}}^{\text {split }}(\widetilde{R}, \widetilde{I})$. (See Section 2.2.) Let $\widetilde{K}:=\operatorname{Frac}(\widetilde{R})$ and $\widetilde{\eta}:=\operatorname{Spec}(\widetilde{K})$. Let $\left(\widetilde{B}, \lambda_{\widetilde{B}}, i_{\widetilde{B}}, X, Y, \phi, \widetilde{c}, \widetilde{c}^{\vee}\right)$ be the pullback of $\left(B, \lambda_{B}, i_{B}, X, Y, \phi, c, c^{\vee}\right)$ to $\widetilde{R}$, and let $\widetilde{\tau}: \mathbf{1}_{Y \times X, \widetilde{\eta}} \stackrel{\sim}{\rightarrow}\left(c^{\vee} \times c\right)^{*} \mathcal{P}_{B, \widetilde{\eta}}^{\otimes-1}$ be the pullback of $\breve{\tau}$ to $\widetilde{\eta}$. Then we obtain a tautological tuple $\left(\widetilde{B}, \lambda_{\widetilde{B}}, i_{\widetilde{B}}, X, Y, \phi, \widetilde{c}, \widetilde{c} \vee, \widetilde{\tau}\right)$ over $\widetilde{R}$, which defines an object in $\operatorname{DD}_{\mathrm{PE}, \mathcal{O}}^{\text {split }}(\widetilde{R}, \widetilde{I})$, and hence an object $(\widetilde{A}, \widetilde{\lambda}, \widetilde{i})$ of $\operatorname{DEG}_{\mathrm{PE}, \mathcal{O}}^{\text {split }}(\widetilde{R}, \widetilde{I})$ by Mumford's construction $\mathrm{M}_{\mathrm{PE}, \mathcal{O}}^{\text {split }}(\widetilde{R}, \widetilde{I})$.

Let $S_{1}$ be the maximal dense subscheme of $\operatorname{Spec}(\widetilde{R} \underset{\mathbb{Z}}{\otimes} \mathbb{Q})$ over which $\widetilde{A}$ is an abelian scheme. By [4, Ch. III, Proposition 5.10 and Corollary 5.11] (or [10, Proposition 
4.5.3.10 and Corollary 4.5.3.11]), $\widetilde{\tau}: \mathbf{1}_{Y \times X, \widetilde{\eta}} \stackrel{\sim}{\rightarrow}\left(c^{\vee} \times c\right)^{*} \mathcal{P}_{B, \widetilde{\eta}}^{\otimes-1}$ extends to a trivialization of biextensions $\tau_{S_{1}}: \mathbf{1}_{Y \times X, S_{1}} \stackrel{\sim}{\rightarrow}\left(c^{\vee} \times c\right)^{*} \mathcal{P}_{B, S_{1}}^{\otimes}$. By the above construction, there is a morphism from $S^{\circ}=\operatorname{Spec}\left(R^{\circ}\right)$ to $\operatorname{Spec}(\widetilde{R} \underset{\mathbb{Q}}{\otimes})$, mapping the generic point $\eta^{\circ}=\operatorname{Spec}\left(K^{\circ}\right)$ to $S_{1}$, such that the pullback of the tuple $\left(\widetilde{B}, \lambda_{\widetilde{B}}, i_{\widetilde{B}}, X, Y, \phi, \widetilde{c}, \widetilde{c}^{\vee}, \tau_{S_{1}}\right)$ to $\operatorname{Spec}\left(R^{\circ}\right)$ is isomorphic to $\left(B^{\circ}, \lambda_{B^{\circ}}, i_{B^{\circ}}, X, Y, \phi, c^{\circ}, c^{\circ \vee}, \tau^{\circ}\right)$. By functoriality of Mumford's construction, this shows that the pullbacks of $(\widetilde{A}, \widetilde{\lambda}, \widetilde{i}) \rightarrow \operatorname{Spec}(\widetilde{R})$ and $\left(A^{\circ}, \lambda^{\circ}, i^{\circ}\right) \rightarrow \operatorname{Spec}\left(R^{\circ}\right)$ to $\operatorname{Spec}\left(K^{\circ}\right)$ are isomorphic to each other. Let $\left(K^{\circ}\right)^{\text {sep }}$ denote a separable closure of $K^{\circ}$. Let $S_{1}^{\prime} \rightarrow S_{1}$ be a finite étale morphism from an integral scheme, such that the composition of canonical morphisms $\operatorname{Spec}\left(\left(K^{\circ}\right)^{\mathrm{sep}}\right) \rightarrow$ $\operatorname{Spec}\left(K^{\circ}\right) \rightarrow S_{1}$ lifts to a canonical morphism $\operatorname{Spec}\left(\left(K^{\circ}\right)^{\text {sep }}\right) \rightarrow S_{1}^{\prime}$ (matching pullbacks of all tautological data). Then, as in the second paragraph of the proof of Theorem 4.1, the canonical morphism $S_{1}^{\prime} \rightarrow \mathrm{M}_{\mathcal{H}}$ factors through $S_{1}^{\prime} \rightarrow \mathrm{Sh}_{\mathcal{H}, \text { can }} \hookrightarrow \mathrm{M}_{\mathcal{H}}$.

5.3. Ends of proofs. Let $R^{\nabla}$ be a mixed characteristic discrete valuation ring of residue characteristic $p$, with a morphism $\operatorname{Spec}\left(R^{\nabla}\right) \rightarrow \operatorname{Spec}(\widetilde{R})$ whose restriction to the generic point factors through $S_{1}^{\prime} \rightarrow \operatorname{Spec}(\widetilde{R})$ and whose restriction to the special point factors through $\operatorname{Spec}(\widetilde{R} / \widetilde{I}) \rightarrow \operatorname{Spec}(\widetilde{R})$. Such an $R^{\nabla}$ exists because $\operatorname{Spec}(\widetilde{R})$ is noetherian and integral. Since the pullback of $\widetilde{A}$ to $\operatorname{Spec}(\widetilde{R} / \widetilde{I})$ is the extension of the pullback of the abelian scheme $B$ by the torus with constant character group $X$, the pullback of $\widetilde{A}$ to $\operatorname{Spec}\left(R^{\nabla}\right)$ is a degeneration of $\operatorname{rank} \operatorname{rk}_{\mathbb{Z}} X=r_{0}$ based at $\mathrm{Sh}_{\mathcal{H} \text {,can }}$, showing that $r_{0} \leq r_{p}$. This completes the proof of Theorem 5.1.

Now Theorem 1.2 follows as a combination of Theorems 4.1 and 5.1.

The relations among the base schemes involved in the proof of Theorem 5.1 can be summarized in the following commutative diagram (cf. the diagram following the proof of Theorem 4.1), in which the dotted arrows are induced by the solid arrows by the connectedness of $S_{1}^{\prime}$ :

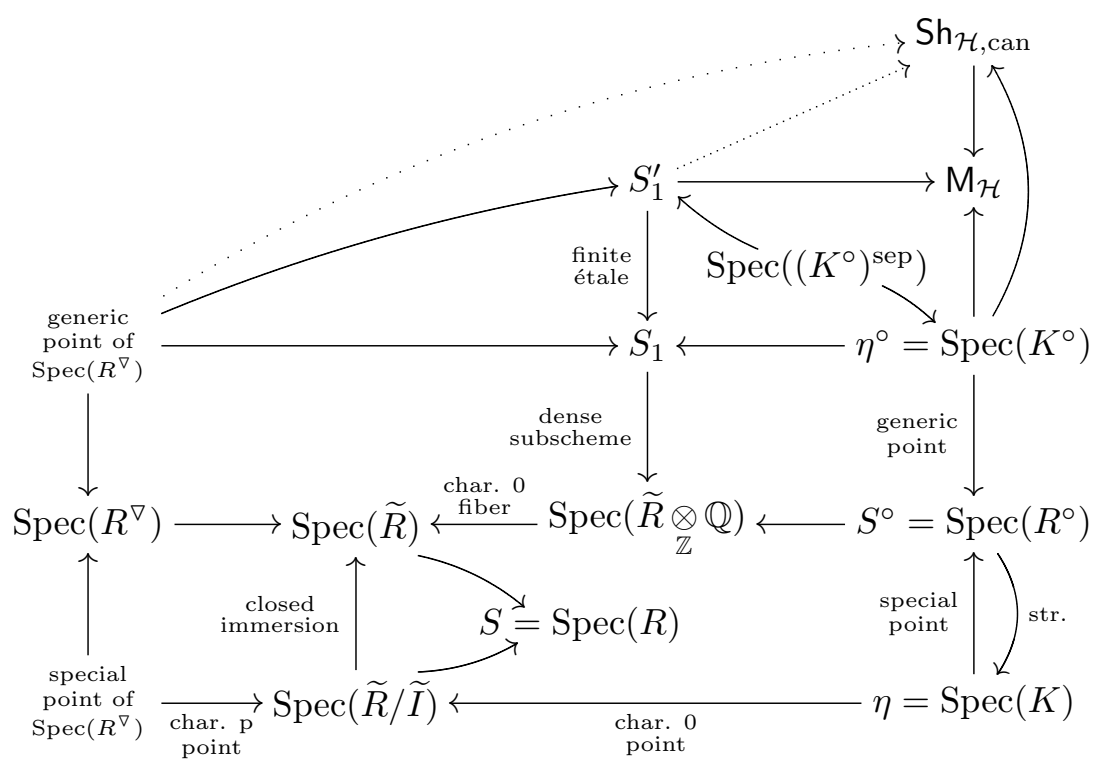


(The arrows from $\operatorname{Spec}(\widetilde{R})$ and $\operatorname{Spec}(\widetilde{R} / \widetilde{I})$ to $S=\operatorname{Spec}(R)$ exist because of their construction; cf. the commutative diagram in Section 3.4.)

\section{Acknowledgments}

We thank Sug Woo Shin and Adrian Vasiu for asking us to explain special cases of Theorem 4.9 using the theory of degeneration of abelian varieties with PEL structures. Special thanks must be given to Adrian Vasiu for his thought-provoking questions. We thank Keerthi Madapusi, Junecue Suh, and Yichao Tian for their comments on earlier versions of this article, and thank Ching-Li Chai and Teruyoshi Yoshida for suggestions on our presentation. We thank Dong Uk Lee for informing us his work on Morita's conjecture (based on $[14,16]$ ) when we first announced our result in July 2009. Finally, we thank the referee for the careful reading and many helpful comments and suggestions.

The author is supported by the Qiu Shi Science and Technology Foundation, and by the National Science Foundation under agreement No. DMS-0635607. Any opinions, findings, and conclusions or recommendations expressed in this material are those of the author and do not necessarily reflect the views of these organizations.

\section{References}

[1] A. Ash, D. Mumford, M. Rapoport and Y. Tai, Smooth compactification of locally symmetric varieties, in 'Lie groups: history frontiers and applications', 4, Math Sci Press, Brookline, MA, 1975.

[2] W. L. Baily, Jr. and A. Borel, Compactification of arithmetic quotients of bounded symmetric domains, Ann. Math. (2) 84(3) (1966), 442-528.

[3] A. Borel and Harish-Chandra, Arithmetic subgroups of algebraic groups, Ann. Math. (2) 75(3) (1962), 483-535.

[4] G. Faltings and C.-L. Chai, Degeneration of Abelian varieties, in 'Ergebnisse der Mathematik und ihrer Grenzgebiete, 3. Folge', 22, Springer-Verlag, Berlin, Heidelberg, New York, 1990.

[5] A. Grothendieck, ed., Groupes de monodromie en géométrie algébrique (SGA 7 I), in 'Lecture Notes in Mathematics', 288, Springer-Verlag, Berlin, Heidelberg, New York, 1972.

[6] A. Grothendieck and J. Dieudonné, Eléments de géométrie algébrique, in 'Publications mathématiques de l'I.H.E.S.', 4, 8, 11, 17, 20, 24, 28, 32, Institut des Hautes Etudes Scientifiques, Paris, 1960, 1961, 1961, 1963, 1964, 1965, 1966, 1967.

[7] M. Harris, Functorial properties of toroidal compactifications of locally symmetric varieties, Proc. London Math. Soc. (3) 59 (1989) 1-22.

[8] G. Kempf, F. Knudsen, D. Mumford and B. Saint-Donat, Toroidal embeddings I, in 'Lecture Notes in Mathematics', 339, Springer-Verlag, Berlin, Heidelberg, New York, 1973.

[9] R. E. Kottwitz, Points on some Shimura varieties over finite fields, J. Amer. Math. Soc. 5(2) (1992), 373-444.

[10] K.-W. Lan, Arithmetic compactification of PEL-type Shimura varieties, Ph.D. Thesis, Harvard University, Cambridge, Massachusetts, 2008, Errata and revision available online at the author's website.

[11] - Comparison between analytic and algebraic constructions of toroidal compactifications of PEL-type Shimura varieties, J. Reine Angew. Math., in press. DOI: 10.1515/CRELLE. 2011.099.

[12] Y. Morita, On potential good reduction of abelain varieties, J. Fac. Univ. Tokyo. Sect. IA Math. 22(3) (1975), 437-447.

[13] D. Mumford, J. Fogarty and F. Kirwan, Geometric Invariant Theory, in 'Ergebnisse der Mathematik und ihrer Grenzgebiete', 34, Springer-Verlag, Berlin, Heidelberg, New York, 3rd edition, 1994. 
[14] F. Paugam, Galois representations, Mumford-Tate groups and good reduction of abelian varieties, Math. Ann. 329(1) (2004), 119-160.

[15] R. Pink, Arithmetic compactification of mixed Shimura varieties, Ph.D. thesis, Rheinischen Friedrich-Wilhelms-Universität, Bonn, 1989.

[16] A. Vasiu, Projective integral models of Shimura varieties of Hodge type with compact factors, J. Reine Angew. Math. 618 (2008), 51-75.

Department of Mathematics, Princeton University, and School of Mathematics, InstiTute For Advanced Study, Princeton, NJ 08544, USA

E-mail address: klan@math.princeton.edu 
